\title{
On the Matchings-Jack conjecture for Jack connection coefficients indexed by two single part partitions
}

\author{
Andrei L. Kanunnikov \\ Department of Higher Algebra \\ Moscow State University \\ Moscow, Russia. \\ andrew . kanunnikov@gmail . com
}

\author{
Ekaterina A. Vassilieva \\ LIX \\ Ecole Polytechnique \\ Palaiseau, France. \\ ekaterina.vassilieva@lix.polytechnique.fr
}

Submitted: Mar 7, 2015; Accepted: Mar 4, 2016; Published: Mar 18, 2016

Mathematics Subject Classifications: 05E05, 05A19

\begin{abstract}
This article is devoted to the study of Jack connection coefficients, a generalization of the connection coefficients of the classical commutative subalgebras of the group algebra of the symmetric group closely related to the theory of Jack symmetric functions. First introduced by Goulden and Jackson (1996) these numbers indexed by three partitions of a given integer $n$ and the Jack parameter $\alpha$ are defined as the coefficients in the power sum expansion of some Cauchy sum for Jack symmetric functions. Goulden and Jackson conjectured that they are polynomials in $\beta=\alpha-1$ with non negative integer coefficients of combinatorial significance, the Matchings-Jack conjecture.

In this paper we look at the case when two of the integer partitions are equal to the single part $(n)$. We use an algebraic framework of Lassalle (2008) for Jack symmetric functions and a bijective construction in order to show that the coefficients satisfy a simple recurrence formula and prove the Matchings-Jack conjecture in this case. Furthermore we exhibit the polynomial properties of more general coefficients where the two single part partitions are replaced by an arbitrary number of integer partitions either equal to $(n)$ or $\left[1^{n-2} 2\right]$.
\end{abstract}

\section{Introduction}

\subsection{Integer partitions}

For any integer $n$ we denote $[n]=\{1, \ldots, n\}, S_{n}$ the symmetric group on $n$ elements and $\lambda=\left(\lambda_{1}, \lambda_{2}, \ldots, \lambda_{p}\right) \vdash n$ an integer partition of $|\lambda|=n$ with $\ell(\lambda)=p$ parts sorted in decreasing order. If $m_{i}(\lambda)$ is the number of parts of $\lambda$ that are equal to $i$, then we may write $\lambda$ as $\left[1^{m_{1}(\lambda)} 2^{m_{2}(\lambda)} \ldots.\right]$ and define $z_{\lambda}=\prod_{i} i^{m_{i}(\lambda)} m_{i}(\lambda)$ !. A partition $\lambda$ is usually represented as a Young diagram of $|\lambda|$ boxes arranged in $\ell(\lambda)$ lines so that the $i$-th line contains $\lambda_{i}$ 
boxes. Given a box $s$ in the diagram of $\lambda$, let $l^{\prime}(s), l(s), a(s), a^{\prime}(s)$ be the number of boxes to the north, south, east, west of $s$ respectively. These statistics are called co-leglength, leglength, armlength, co-armlength respectively. As an example the box $s$ in the following diagram representing $\lambda=(6,4,2,1,1)$ verifies $l^{\prime}(s)=1, l(s)=0, a(s)=1$ and $a^{\prime}(s)=2$.

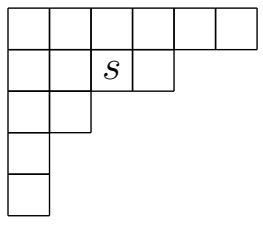

We define for some parameter $\alpha$ :

$$
h_{\lambda}(\alpha)=\prod_{s \in \lambda}(\alpha a(s)+l(s)+1), \quad h_{\lambda}^{\prime}(\alpha)=\prod_{s \in \lambda}(\alpha(1+a(s))+l(s)),
$$

and denote $j_{\lambda}(\alpha)$ the product

$$
j_{\lambda}(\alpha)=h_{\lambda}(\alpha) h_{\lambda}^{\prime}(\alpha) .
$$

Finally we adopt the following notations consistent with [23] for operations on integer partitions. For a partition $\lambda$ containing at least one part $k$ denote $\lambda_{\downarrow}(k)$ the partition obtained from $\lambda$ by removing one of the parts $k$ and adding a part $k-1$ and $\lambda^{\uparrow(k)}$ the partition obtained from $\lambda$ by removing one of the parts $k$ and adding a part $k+1$. If $\lambda$ contains a part $k$ and a part $l$ we denote $\lambda_{\downarrow}(k, l)$ the partition obtained from $\lambda$ by removing a part $k$ and a part $l$ and adding a part $k+l-1$. Finally if $\lambda$ contains a part $k+l+1$ we denote $\lambda^{\uparrow(k, l)}$ the partition obtained from $\lambda$ by adding a part $k$ and a part $l$ and removing a part $k+l+1$. So we denote

$$
\begin{array}{llrl}
\lambda_{\downarrow(k)} & =\lambda \backslash k \cup(k-1), & & \lambda_{\downarrow(k, l)}=\lambda \backslash(k, l) \cup(k+l-1), \\
\lambda^{\uparrow(k)}=\lambda \backslash k \cup(k+1), & & \lambda^{\uparrow(k, l)}=\lambda \backslash(k+l+1) \cup(k, l) .
\end{array}
$$

\subsection{Classes of permutations indexed by partitions}

The conjugacy classes of the symmetric group $S_{n}$ are indexed by partitions of $n$ according to the cycle type of the permutations they contain. For $\lambda \vdash n$ we denote $C_{\lambda}$ the class of permutations of cycle type $\lambda$. The cardinality of the conjugacy classes is given by $\left|C_{\lambda}\right|=n ! / z_{\lambda}$. We look at matchings of the set $[n] \cup[\widehat{n}]=\{1, \ldots n, \widehat{1}, \ldots, \widehat{n}\}$ which we view as fixpoint-free involutions in $S_{2 n}$. Note that for $f, g$ fixpoint-free involutions of $S_{2 n}$, the disjoint cycles of the product $f \circ g$ have repeated lengths i.e. $f \circ g \in C_{\lambda \lambda}$ for some $\lambda \vdash n$. We consider the hyperoctahedral group $B_{n}$ as the centralizer of $f_{\star}=(1 \widehat{1})(2 \widehat{2}) \cdots(n \widehat{n})$ in $S_{2 n}$. As shown in e.g. [25, VII.2] the double cosets of $B_{n}$ in $S_{2 n}$ are also indexed by integer partitions of $n$. We denote by $K_{\lambda}$ the double coset indexed by $\lambda \vdash n$ consisting of all the permutations $\omega$ of $S_{2 n}$ such that $f_{\star} \circ \omega \circ f_{\star} \circ \omega^{-1}$ belongs to $C_{\lambda \lambda}$. According to this definition $K_{\lambda}=B_{n} \omega B_{n}$ for any $\omega$ in $K_{\lambda}$ and, in particular, $B_{n}=K_{\left[1^{n}\right]}$. We have [25, VII.2, (2.3)] $\left|B_{n}\right|=2^{n} n$ ! and $\left|K_{\lambda}\right|=\left|B_{n}\right|^{2} /\left(2^{\ell(\lambda)} z_{\lambda}\right)$. 


\subsection{Symmetric functions}

Let $\Lambda$ be the ring of symmetric functions. Denote $m_{\lambda}(x)$ the monomial symmetric function indexed by $\lambda$ on $x=\left(x_{1}, x_{2}, \ldots\right), p_{\lambda}(x)$ and $s_{\lambda}(x)$ the power sum and Schur symmetric functions respectively. Whenever the indeterminate is not relevant we shall simply write $m_{\lambda}, p_{\lambda}$ and $s_{\lambda}$. Let $\langle\cdot, \cdot\rangle$ be the scalar product on $\Lambda$ such that the power sum symmetric functions verify $\left\langle p_{\lambda}, p_{\mu}\right\rangle=z_{\lambda} \delta_{\lambda \mu}$ where $\delta_{\lambda \mu}$ is the Kronecker delta. The Schur symmetric functions $s_{\lambda}$ are characterized by the fact that they form an orthogonal basis (they form even an orthonormal basis) of $\Lambda$ for $\langle\cdot, \cdot\rangle$ and the transition matrix between Schur and monomial symmetric functions is upper triangular.

The zonal polynomials $Z_{\lambda}$ constitute another important basis of $\Lambda$ directly linked with the theory of the zonal spherical functions. Zonal polynomials verify the same properties as the $s_{\lambda}$ (except for the unit length property) if the scalar product is replaced by $\langle\cdot, \cdot\rangle_{2}$ with $\left\langle p_{\lambda}, p_{\mu}\right\rangle_{2}=2^{\ell(\lambda)} z_{\lambda} \delta_{\lambda \mu}$. In the general case, using an additional parameter $\alpha$, Henry Jack [15] introduced the bases of Jack symmetric functions $J_{\lambda}^{\alpha}$ orthogonal for the alternative scalar product $\langle\cdot, \cdot\rangle_{\alpha}$ defined by $\left\langle p_{\lambda}, p_{\mu}\right\rangle_{\alpha}=\alpha^{\ell(\lambda)} z_{\lambda} \delta_{\lambda \mu}$. We use the normalization of Jack symmetric functions such that $\left[m_{\lambda}\right] J_{\lambda}^{\alpha}=h_{\lambda}(\alpha)$. As a result we have

$$
J_{\lambda}^{1}=h_{\lambda}(1) s_{\lambda} \quad \text { and } \quad J_{\lambda}^{2}=Z_{\lambda} .
$$

Moreover, as shown by Stanley in [33],

$$
\left\langle J_{\lambda}^{\alpha}, J_{\mu}^{\alpha}\right\rangle_{\alpha}=j_{\lambda}(\alpha) \delta_{\lambda \mu}
$$

\subsection{Classical connection coefficients and their combinatorial interpretation}

By abuse of notation let $C_{\lambda}$ (resp. $K_{\lambda}$ ) also represent the formal sum of its elements in the group algebra $\mathbb{C} S_{n}$ (resp. $\mathbb{C} S_{2 n}$ ). So $\left\{C_{\lambda} \mid \lambda \vdash n\right\}$ forms a basis of the class algebra i.e. the center of $\mathbb{C} S_{n}$ and $\left\{K_{\lambda} \mid \lambda \vdash n\right\}$ forms a basis of the double coset algebra i.e. the commutative subalgebra of $\mathbb{C} S_{2 n}$ identified as the Hecke algebra of the Gelfand pair $\left(S_{2 n}, B_{n}\right)$. For integer $s \geqslant 2$ and partitions $\lambda^{1}, \ldots, \lambda^{s} \vdash n$, we define the connection coefficients of the class algebra $c_{\lambda^{2}, \ldots, \lambda^{s}}^{\lambda^{1}}$ and the connection coefficients of the double coset algebra $b_{\lambda^{2}, \ldots, \lambda^{s}}^{\lambda^{1}}$ by

$$
c_{\lambda^{2}, \ldots, \lambda^{s}}^{\lambda^{1}}=\left[C_{\lambda^{1}}\right] \prod_{i \geqslant 2} C_{\lambda^{i}}, \quad b_{\lambda^{2}, \ldots, \lambda^{s}}^{\lambda^{1}}=\left[K_{\lambda^{1}}\right] \prod_{i \geqslant 2} K_{\lambda^{i}}
$$

From a combinatorial point of view $c_{\lambda^{2}, \ldots, \lambda^{s}}^{\lambda^{1}}$ (resp. $b_{\lambda^{2}, \ldots, \lambda^{s}}^{\lambda^{1}}$ ) is the number of ways to write a given permutation $\sigma_{1}$ of $C_{\lambda^{1}}$ (resp. $K_{\lambda^{1}}$ ) as the ordered product of $s-1$ permutations $\sigma_{2} \circ \ldots \circ \sigma_{s}$ where $\sigma_{i}$ is in $C_{\lambda^{i}}$ (resp. $K_{\lambda^{i}}$ ). When $s=3$, the coefficients $c_{\mu \nu}^{\lambda}$ and $b_{\mu \nu}^{\lambda}$ also admit a nice interpretation in terms of graphs on $2 n$ vertices (see e.g. [8], [13]). For a given partition $\lambda=\left(\lambda_{1}, \ldots, \lambda_{p}\right)$ of integer $n$, consider the graph $G$ on $2 n$ vertices consisting of $p$ cycles of lengths $2 \lambda_{1}, \ldots, 2 \lambda_{p}$. A matching in $G$ is a set of edges without common vertices that contains all the vertices of $G$. Coloring successively the edges of the cycles of $G$ in gray and black colors, we get two matchings: g (gray edges) and $\mathbf{b}$ (black edges). We call 
such a two-colour graph induced by $\lambda$ a $\lambda$-graph. Label the vertices of $G$ by $[n] \cup[\widehat{n}]$ such that edges $\{i, \hat{i}\}$ are gray and the vertices of the $i$-th cycle are successively labelled

$$
\sum_{j=1}^{i-1} \lambda_{j}+1, \widehat{\sum_{j=1}^{i-1} \lambda_{j}}+1, \sum_{j=1}^{i-1} \lambda_{j}+2, \widehat{\sum_{j=1}^{i-1} \lambda_{j}}+2, \ldots, \sum_{j=1}^{i} \lambda_{j}, \widehat{\sum_{j=1}^{i} \lambda_{j}} .
$$

This labeling is named canonical. A matching in which all edges are of kind $\{i, \widehat{j}\}$ is called bipartite.

Example 1. Figure 1 depicts a canonically labeled $\lambda$-graph for $\lambda=(3,2,2,1)$.
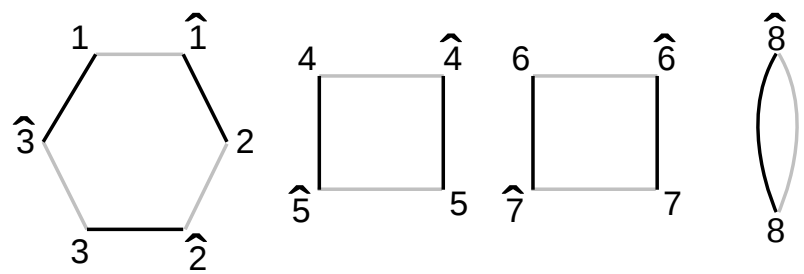

Figure 1: The union of the black and gray matchings forms the $(3,2,2,1)$-graph.

Denote $\tilde{b}_{\mu \nu}^{\lambda}$ the quantity $\tilde{b}_{\mu \nu}^{\lambda}:=b_{\mu \nu}^{\lambda} /\left|B_{n}\right|$. We have the following proposition.

Proposition 1 ([8], proposition 4.1; [13], Lemma 3.2). Let $\lambda \vdash n$, G be the induced $\lambda$-graph and define the matchings $\mathbf{b}$ and $\mathbf{g}$ in $G$ as above. Then $\tilde{b}_{\mu \nu}^{\lambda}$ (resp. $c_{\mu \nu}^{\lambda}$ ) is the number of matchings (resp. bipartite matchings) $\delta$ such that the graphs $\mathbf{b} \cup \delta$ and $\mathbf{g} \cup \delta$ are respectively a $\mu$-graph and a $\nu$-graph. We denote the set of all such matchings by $\mathcal{G}_{\mu \nu}^{\lambda}$.

We call a matching $\delta$ such that both $\mathbf{b} \cup \delta$ and $\mathbf{g} \cup \delta$ are $2 n$-cycles (i.e. (n)-graphs) a good matching. Denote by $\mathcal{G}(\lambda)\left(=\mathcal{G}_{(n)(n)}^{\lambda}\right)$ the set of all good matchings of the canonically labeled $\lambda$-graph $G$. Due to Proposition 1 ,

$$
\tilde{b}_{n n}^{\lambda}=|\mathcal{G}(\lambda)| \quad \text { and } \quad c_{n n}^{\lambda}=\mid\{\delta \in \mathcal{G}(\lambda) \mid \delta \text { is bipartite }\} \mid .
$$

Example 2. 1. One can see $\lambda$-graphs for $\lambda$ with $|\lambda| \leqslant 2$ on Figure 2. So we have

$$
\begin{array}{lll}
\tilde{b}_{11}^{1}=1, & \tilde{b}_{22}^{2}=1, & \tilde{b}_{22}^{(1,1)}=2, \\
c_{11}^{1}=1, & c_{22}^{2}=0, & c_{22}^{(1,1)}=1 .
\end{array}
$$

2. Figure 3 depicts the 4 good matchings in the case $\lambda=(3)$. Only the leftmost one is bipartite. As a result $\tilde{b}_{33}^{3}=4$ and $c_{33}^{3}=1$. 

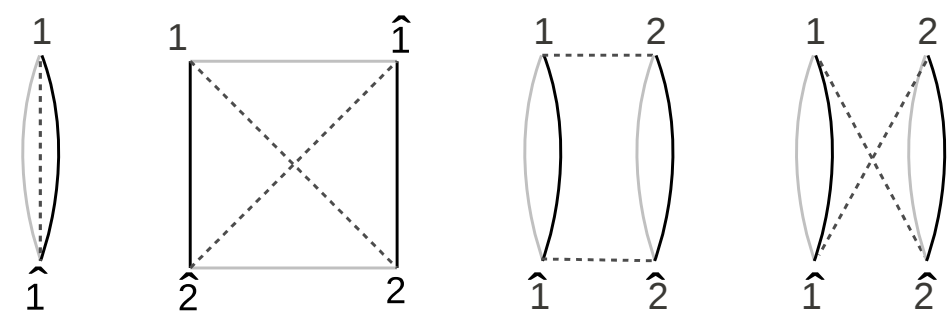

Figure 2: Good matchings for $|\lambda| \leqslant 2$.
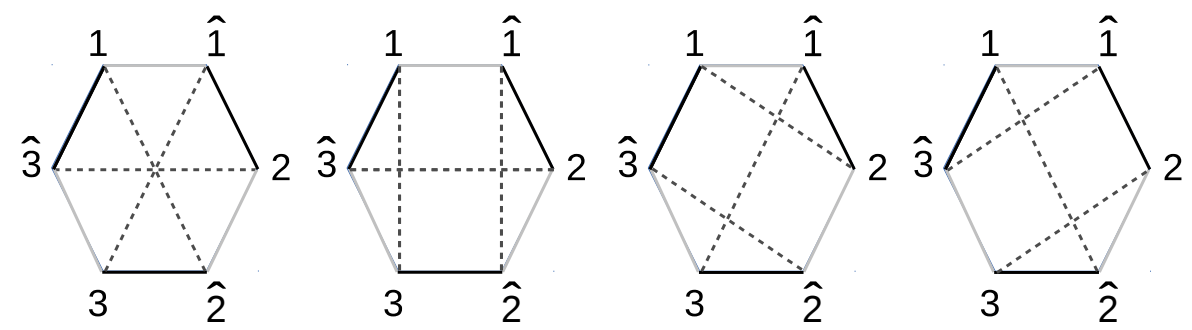

Figure 3: Good matchings for $\lambda=(3)$.

\subsection{Jack connection coefficients and the Matchings-Jack conjecture}

It is easy to show (see e.g. [8], [13], [37]) that connection coefficients are linked to some extended Cauchy sums for Schur and zonal symmetric functions. In particular, one has:

$$
\begin{aligned}
& \sum_{\lambda, \mu, \nu \vdash n} z_{\lambda}^{-1} c_{\mu \nu}^{\lambda} p_{\lambda}(x) p_{\mu}(y) p_{\nu}(z)=\sum_{\gamma \vdash n} h_{\gamma}(1) s_{\gamma}(x) s_{\gamma}(y) s_{\gamma}(z), \\
& \sum_{\lambda, \mu, \nu \vdash n} 2^{-\ell(\lambda)} z_{\lambda}^{-1} \frac{b_{\mu \nu}^{\lambda}}{\left|B_{n}\right|} p_{\lambda}(x) p_{\mu}(y) p_{\nu}(z)=\sum_{\gamma \vdash n} \frac{Z_{\gamma}(x) Z_{\gamma}(y) Z_{\gamma}(z)}{\left\langle Z_{\gamma}, Z_{\gamma}\right\rangle_{2}} .
\end{aligned}
$$

In the general case, Goulden and Jackson [8] considered the coefficients $a_{\mu \nu}^{\lambda}(\alpha)$ in the power sum expansions of similar sums for Jack symmetric functions

$$
\sum_{\lambda, \mu, \nu \vdash n} \alpha^{-\ell(\lambda)} z_{\lambda}^{-1} a_{\mu \nu}^{\lambda}(\alpha) p_{\lambda}(x) p_{\mu}(y) p_{\nu}(z)=\sum_{\gamma \vdash n} \frac{J_{\gamma}^{\alpha}(x) J_{\gamma}^{\alpha}(y) J_{\gamma}^{\alpha}(z)}{\left\langle J_{\gamma}^{\alpha}, J_{\gamma}^{\alpha}\right\rangle_{\alpha}} .
$$

In view of Equations (1), (5), (6) and (7)

$$
a_{\mu \nu}^{\lambda}(1)=c_{\mu \nu}^{\lambda} \quad \text { and } \quad a_{\mu \nu}^{\lambda}(2)=\frac{1}{\left|B_{n}\right|} b_{\mu \nu}^{\lambda}=\tilde{b}_{\mu \nu}^{\lambda} .
$$

Exhaustive computations of $a_{\mu \nu}^{\lambda}(\alpha)$ for all $\lambda, \mu, \nu \vdash n \leqslant 8$ (see [8]) showed that the $a_{\mu \nu}^{\lambda}(\alpha)$ are polynomials in $\beta=\alpha-1$ with non negative integer coefficients and of degree at most 
$n-\min \{\ell(\mu), \ell(\nu)\}$. Goulden and Jackson conjectured this property for arbitrary $\lambda, \mu, \nu$. Moreover, following the combinatorial interpretation in Proposition 1, they also suggested the stronger Matchings-Jack conjecture.

Conjecture 1 ([8], conjecture 4.2). For $\lambda, \mu, \nu \vdash n$ there exists a function $\mathrm{wt}_{\lambda}: \mathcal{G}_{\mu \nu}^{\lambda} \rightarrow$ $\{0,1, \ldots, n-1\}$ (see Proposition 1) such that

$$
a_{\mu \nu}^{\lambda}(\beta+1)=\sum_{\delta \in \mathcal{G}_{\mu \nu}^{\lambda}} \beta^{\operatorname{wt}_{\lambda}(\delta)}
$$

and $\mathrm{wt}_{\lambda}(\delta) \leqslant n-\min \{\ell(\mu), \ell(\nu)\}, \mathrm{wt}_{\lambda}(\delta)=0 \Longleftrightarrow \delta$ is bipartite.

They proved this conjecture in the cases $\lambda=\left[1^{n}\right]$ and $\lambda=\left[1^{n-2} 2^{1}\right]$. Following [37], we use the terminology of Jack connection coefficients for $a_{\mu \nu}^{\lambda}(\alpha)$ (although the normalization in [37] differs from the one of this paper). Our work is mainly devoted to the case $\mu=\nu=(n)$. We show various results for Jack connection coefficients in this case, including the proof of the Matchings-Jack conjecture.

Remark 1. As shown recently in [5, Section 4.2 and Appendix B.1], the coefficients $a_{\mu \nu}^{\lambda}(\alpha)$ with $\lambda, \mu, \nu \vdash n$ are the connection coefficients of the algebra of functions on Young diagrams of size $n$ as they provide the coordinates in the basis of Jack characters (see Section 2.2) of the product of two elements of this basis. As a result, the terminology of Jack connection coefficients is well justified even for $\alpha \notin\{1,2\}$.

\subsection{Main results}

Our proof of Goulden and Jackson's conjecture is based on the recurrence formula (9) which derives from the following theorem proved in Section 3.

For simplicity, we write $n$ instead of $(n)$ in the indices of the coefficients.

Theorem 1. For positive integer $n$ and integer partitions $\nu \vdash n$ and $\lambda \vdash n+1$, the Jack connection coefficients defined in Equation (7) satisfy the following formula:

$$
\begin{aligned}
\sum_{i: m_{i-1}(\nu) \neq 0} i\left(m_{i}(\nu)+1\right) & a_{n+1, \nu^{\uparrow}(i-1)}^{\lambda}(\alpha) \\
& =\sum_{i=1}^{\ell(\lambda)} \lambda_{i}\left[(\alpha-1)\left(\lambda_{i}-1\right) a_{n, \nu}^{\lambda_{\downarrow}\left(\lambda_{i}\right)}(\alpha)\right. \\
& \left.+\sum_{d=1}^{\lambda_{i}-2} a_{n, \nu}^{\lambda^{\uparrow\left(\lambda_{i}-1-d, d\right)}}(\alpha)+\alpha \sum_{j \neq i} \lambda_{j} a_{n, \nu}^{\lambda_{\downarrow}\left(\lambda_{i}, \lambda_{j}\right)}(\alpha)\right] .
\end{aligned}
$$

In the special case $\nu=(n)$ the formula (8) becomes recursive. Besides, as shown in Section 4, the expression in brackets does not depend on $i$. This remarkable property allows us to derive the following theorem. 
Theorem 2. For integer $n$ and partition $\lambda \vdash n+1$, the Jack connection coefficients verify the following recurrence formula for any $i \in\{1, \ldots, \ell(\lambda)\}$ :

$$
\begin{aligned}
a_{n+1, n+1}^{\lambda}(\alpha)=(\alpha-1) & \left(\lambda_{i}-1\right) a_{n n}^{\lambda_{\downarrow}\left(\lambda_{i}\right)}(\alpha) \\
& +\sum_{d=1}^{\lambda_{i}-2} a_{n n}^{\lambda^{\uparrow\left(\lambda_{i}-1-d, d\right)}}(\alpha)+\alpha \sum_{j \neq i} \lambda_{j} a_{n n}^{\lambda_{\downarrow}\left(\lambda_{i}, \lambda_{j}\right)}(\alpha) .
\end{aligned}
$$

Remark 2. One can use the fact that Equation (9) is true for any $i$ to derive additional formulas. For instance, let $\mu$ be a partition of integer $n-1$ and $\lambda=\mu \cup(1)$ a partition of $n$. Setting $i$ such that $\lambda_{i}=1$ in Equation (9) yields the following expression:

$$
a_{n n}^{\mu \cup(1)}(\alpha)=\alpha(n-1) a_{n-1, n-1}^{\mu}(\alpha) .
$$

Iterate this relation for any partition $\lambda$ of $n$ that we write $\lambda=\mu \cup\left(1^{m_{1}(\lambda)}\right)$ :

$$
a_{n n}^{\lambda}(\alpha)=\alpha^{m_{1}(\lambda)} \frac{(n-1) !}{\left(n-m_{1}(\lambda)-1\right) !} a_{n-m_{1}(\lambda), n-m_{1}(\lambda)}^{\mu}(\alpha) .
$$

Equation (9) also proves for $\mu \vdash n-2, \lambda=\mu \cup(2)$ and $\lambda_{i}=2$

$$
a_{n n}^{\mu \cup(2)}(\alpha)=\alpha(\alpha-1)(n-2) a_{n-2, n-2}^{\mu}(\alpha)+\alpha \sum_{j} \mu_{j} a_{n-1, n-1}^{\mu^{\uparrow\left(\mu_{j}\right)}} .
$$

Theorem 2 allows us to prove the Matchings-Jack conjecture in the case $\mu=\nu=(n)$.

Theorem 3. Let $\lambda$ be a partition of $n$ and $G$ a canonically labelled $\lambda$-graph. Then there exists a function $\mathrm{wt}_{\lambda}: \mathcal{G}(\lambda) \rightarrow\{0,1, \ldots, n-1\}$ such that

$$
\begin{aligned}
a_{n n}^{\lambda}(\beta+1) & =\sum_{\delta \in \mathcal{G}(\lambda)} \beta^{\mathrm{wt}_{\lambda}(\delta)}, \\
\operatorname{wt}_{\lambda}(\delta)=0 & \Longleftrightarrow \delta \text { is bipartite. }
\end{aligned}
$$

As a consequence, the quantity $a_{n n}^{\lambda}(\beta+1)$ is a nonnegative integer polynomial in $\beta$ with constant term $c_{n n}^{\lambda}=a_{n n}^{\lambda}(1)$ and sum of coefficients equal to $\tilde{b}_{n n}^{\lambda}=a_{n n}^{\lambda}(2)$. Besides

$$
\left[\beta^{n-1}\right] a_{n n}^{\lambda}(\beta+1)=(n-1) !
$$

All of the results stated previously deal with connection coefficients indexed by three partitions. However, as shown in Equation (3), classical connection coefficients are defined and admit nice combinatorial interpretations for an arbitrary number of partitions. In the following paragraph, we state some additional results about Jack connection coefficients indexed by more than three partitions. 
For a sequence of indeterminates $x^{1}, x^{2}, \ldots, x^{s}$ define the more general Jack connection coefficients $a_{\lambda_{2}, \ldots, \lambda_{s}}^{\lambda^{1}}(\alpha)$ by the formula

$$
\sum_{\lambda^{i} \vdash n} \alpha^{-\ell\left(\lambda^{1}\right)} z_{\lambda^{1}}^{-1} a_{\lambda_{2}, \ldots, \lambda_{s}}^{\lambda^{1}}(\alpha) \prod_{i} p_{\lambda^{i}}\left(x^{i}\right)=\sum_{\gamma \vdash n} \frac{\prod_{i} J_{\gamma}^{\alpha}\left(x^{i}\right)}{\left\langle J_{\gamma}^{\alpha}, J_{\gamma}^{\alpha}\right\rangle_{\alpha}}
$$

and denote for any integer partition $\lambda \vdash n$ and integers $l, r \geqslant 0$

$$
a_{\lambda}^{l, r}(\alpha)=a_{(\underbrace{\lambda}_{l}, \ldots,(n)}^{(n), \underbrace{\left[1^{n-2} 2\right], \ldots,\left[1^{n-2} 2\right]}_{r}}(\alpha) .
$$

We show some polynomial properties for the coefficients $a_{\lambda}^{l, r}(\alpha)$.

Theorem 4. Let $D(\alpha)$ denote the Laplace-Beltrami operator (see Equation (20)), $\Delta_{0}(\alpha)=p_{1} / \alpha$ and $\Delta_{k}(\alpha)=\left[D(\alpha), \Delta_{k-1}(\alpha)\right]$. The coefficients $a_{\lambda}^{l, r}(\alpha)$ defined above verify

$$
\alpha^{-\ell(\lambda)}\left|C_{\lambda}\right| a_{\lambda}^{l, r}(\alpha)=\left[p_{\lambda}\right] D(\alpha)^{r} \Delta_{l}(\alpha)^{n-1}\left(p_{1} / \alpha\right)
$$

and for $l \geqslant 2,\left|C_{\lambda}\right| a_{\lambda}^{l, r}(\alpha)$ is a polynomial in $\alpha$ with integer coefficients of degree at most $(n-1)(l-1)+r$. Furthermore, for $0 \leqslant i \leqslant(n-1)(l-1)+r$

$$
\left[\alpha^{i}\right]\left|C_{\lambda}\right| a_{\lambda}^{l, r}(\alpha)=(-1)^{(l-1)(n-1)+r+\ell(\lambda)-1}\left[\alpha^{(l-1)(n-1)+r+\ell(\lambda)-1-i}\right]\left|C_{\lambda}\right| a_{\lambda}^{l, r}(\alpha) .
$$

Using the theory of Jack symmetric functions, we prove Theorem 1 in Section 3. Section 4 is devoted to Theorem 2 and the Matchings-Jack conjecture of Theorem 3. We first provide an algebraic proof of Theorem 2 for general $\alpha$ and, then, reprove the same theorem in the special cases $\alpha=1,2$ thanks to a bijective construction. Using this bijection and the formula for any $\alpha$ we show the Matchings-Jack conjecture. The additional results of Theorem 4 are shown in Section 5 .

\section{Background}

Our work is mainly related to two areas of combinatorics, namely the computation of classical connection coefficients of group algebra of the symmetric group and the study of the coefficients in the power sum expansions of Jack symmetric functions. Relevant background is described in the two following subsections. Although most of this paper uses the interpretation of connection coefficients in terms of graphs and matchings, these coefficients also admit an alternative description in terms of maps. The link between the two interpretations is emphasised in a third subsection.

\subsection{Computation of connection coefficients}

Except for special cases no closed formulas are known for the coefficients $c_{\lambda^{2}, \ldots, \lambda^{s}}^{\lambda^{1}}$ and $b_{\lambda^{2}, \ldots, \lambda^{s}}^{\lambda^{1}}$. Among the early results, when $\lambda^{2}=\lambda^{3}=\ldots \lambda^{s}=\left[1^{n-2} 2\right]$ for some integer $n$, 
the numbers $c_{\lambda^{2}, \ldots, \lambda^{s}}^{\lambda^{1}}$ reduce to the Hurwitz numbers. In [14], a formula is provided for the case $s-1=\left|\lambda^{1}\right|-\ell\left(\lambda^{1}\right)$. More recently, using an inductive argument Bédard and Goupil [1] first found a formula for $c_{\lambda \mu}^{n}$ in the case $\ell(\lambda)+\ell(\mu)=n+1$, which was later reproved by Goulden and Jackson [7] via a bijection with a set of ordered rooted bicolored trees. Later, using characters of the symmetric group and a combinatorial development, Goupil and Schaeffer [9] derived an expression for connection coefficients of arbitrary genus as a sum of positive terms (see Biane [2] for a succinct algebraic derivation; and Poulalhon and Schaeffer [30], and Irving [10] for further generalizations). Closed form formulas of the expansion of the generating series for the $c_{\lambda^{2}, \ldots, \lambda^{s}}^{(n)}$ (for general $s$ ) and $b_{\lambda \mu}^{(n)}$ in the monomial basis were provided by Morales and Vassilieva and Vassilieva in [27], [28], [35] and [36]. Papers [28] and [36] use the topological interpretation of $c_{\lambda^{2}, \ldots, \lambda^{s}}^{(n)}$ and $b_{\lambda \mu}^{(n)}$ in terms of unicellular locally orientable hypermaps and constellations of given vertex degree distribution. Jackson ([16]) computed a general expression for the generating series of the $\sum_{\ell\left(\lambda_{i}\right)=p_{i}} c_{\lambda^{2}, \ldots, \lambda^{s}}^{\lambda^{1}}$ in terms of some explicit polynomials.

\subsection{Jack characters and prior results on the Matchings-Jack conjecture}

The link between Schur (resp. zonal) polynomials and irreducible characters of the symmetric group (resp. zonal spherical functions) is given by the decomposition of the $s_{\lambda}$ (resp. $Z_{\lambda}$ ) in the power sum basis

$$
\begin{aligned}
& s_{\lambda}=\sum_{\mu \vdash n} z_{\mu}^{-1} \chi_{\mu}^{\lambda} p_{\mu}, \\
& Z_{\lambda}=\frac{1}{\left|B_{n}\right|} \sum_{\mu \vdash n} \varphi_{\mu}^{\lambda} p_{\mu}
\end{aligned}
$$

where $\chi_{\mu}^{\lambda}$ is the value of the irreducible character of the symmetric group $\chi^{\lambda}$ indexed by integer partition $\lambda$ at any element of $C_{\mu}$ and the value of the zonal spherical function indexed by $\lambda$ of the Gelfand pair $\left(S_{2 n}, B_{n}\right)$ at the elements of the double coset $K_{\mu}$ is given by $\left|K_{\mu}\right|^{-1} \varphi_{\mu}^{\lambda}$. Using the fact that both Schur and zonal symmetric functions are special cases of Jack symmetric functions, it is natural to focus on a more general form of Equations (14) and (15). Formally, let $\theta_{\mu}^{\lambda}(\alpha)$ denote the coefficient of $p_{\mu}$ in the expansion of $J_{\lambda}^{\alpha}$ in the power sum basis:

$$
J_{\lambda}^{\alpha}=\sum_{\mu} \theta_{\mu}^{\lambda}(\alpha) p_{\mu}
$$

According to Equations (14) and (15), up to a normalization factor, the $\theta_{\mu}^{\lambda}(\alpha)$ 's coincide with the irreducible characters of the symmetric group and the zonal spherical functions in the cases $\alpha=1$ and $\alpha=2$. In the general case Dołęga and Féray [4] named them Jack characters.

The coefficients in the power sum expansion of Jack symmetric functions have received significant attention over the past decades. As an example, Hanlon conjectured a first combinatorial interpretation for them in [11] in terms of digraphs. Stanley proved various 
results for these coefficients in [33]. For instance for simple values of $\mu$ the following formulas are fulfilled for any integer partition $\lambda \vdash n$ :

$$
\begin{aligned}
& \theta_{\left[1^{n}\right]}^{\lambda}(\alpha)=1, \\
& \theta_{\left[1^{n-2} 2^{1}\right]}^{\lambda}(\alpha)=\sum_{s \in \lambda}\left(\alpha a^{\prime}(s)-l^{\prime}(s)\right), \\
& \theta_{(n)}^{\lambda}(\alpha)=\prod_{s \in \lambda \backslash\{(1,1)\}}\left(\alpha a^{\prime}(s)-l^{\prime}(s)\right) .
\end{aligned}
$$

More recent works by Lassalle (see e.g. [23] and [24]) reconsidered these coefficients as generalizations of irreducible characters of the symmetric group and conjectured various polynomial properties in $\alpha$ for the $\theta_{\mu}^{\lambda}(\alpha)$. Furthermore, Lassalle showed in [23] some recursive formulas for $\vartheta_{\mu}^{\lambda}=z_{\mu} \theta_{\mu, 1^{n-k}}^{\lambda}(\alpha)$ where $\lambda \vdash n$ and $\mu \vdash k$ with $m_{1}(\mu)=0$. Namely,

$$
\begin{aligned}
& \sum_{r, s} r s\left(m_{r}(\mu)\left(m_{s}(\mu)-\delta_{r s}\right) \vartheta_{\mu_{\downarrow}(r, s)}^{\lambda}+(\alpha-1) \sum_{r}^{\bullet} r(r-1) \vartheta_{\mu_{\downarrow}(r)}^{\lambda}+\right. \\
& \alpha \sum_{r}^{\bullet} r m_{r}(\mu) \sum_{i=1}^{r-2} \vartheta_{\mu^{\uparrow(i, r-i-1)}}^{\lambda}=-(n+k) \vartheta_{\mu}^{\lambda}+\alpha \sum_{i=1}^{\ell(\lambda)+1} c_{i}(\lambda)\left(\lambda_{i}-(i-1) / \alpha\right)^{2} \vartheta_{\mu}^{\lambda^{(i)}}
\end{aligned}
$$

where the symbol $\sum^{\bullet}$ indicates some additional factors in limit conditions (see [23]), the numbers $c_{i}(\lambda)$ are defined in Equation $(22)$ and $\lambda^{(i)}$ is defined in Section 3.1.

While the formulas in Theorems 1 and 2 share some similarities with the above recurrence formula (one can notice the same operations on partitions on the left-hand side as in Theorem 2) they cannot be derived from it.

Lapointe and Vinet [19] proved that Jack characters are polynomials in $\alpha$ with integer coefficients. Later on Knop and Sahi [17] showed with a different method that these integer coefficients are non negative. (To be more accurate both papers deal with the coefficients in the monomial expansion of the Jack symmetric functions but the implication for the $\theta_{\mu}^{\lambda}(\alpha)$ is immediate). Dołęga and Féray [4] and Dołęga, Féray and Śniady [6] conjectured an expression involving a measure of "non-orientability" of locally orientable hypermaps (an expression also introduced in [8].)

Dołęga and Féray also proved in [5] that the $a_{\mu \nu}^{\lambda}(\alpha)$ are polynomials in $\alpha$ with rational coefficients. Prior to their result, Brown and Jackson in [3] built a candidate object to prove a variant of the Matchings-Jack conjecture also stated in [8]. Although they do not give a formal proof of their solution, evidences supporting their conjecture are provided. In [18], Lacroix focuses on the same variant of the Matchings-Jack conjecture, proves a special case and establishes connections with some combinatorial properties of maps.

Remark 3. Using Equations (16) and (2), one can reformulate Jack connection coefficients as

$$
a_{\lambda_{2}, \ldots, \lambda_{s}}^{\lambda^{1}}(\alpha)=\alpha^{\ell\left(\lambda^{1}\right)} z_{\lambda^{1}} \sum_{\gamma \vdash n} \frac{\prod_{i} \theta_{\lambda^{i}}^{\gamma}(\alpha)}{j_{\gamma}(\alpha)} .
$$

We use this formulation in the following sections. 


\subsection{Maps interpretation}

From a topological point of view, a locally orientable hypermap can be defined as a connected bipartite graph with black and white vertices. Each edge is composed of two half-edges both connecting the two incident vertices. This graph is embedded in a locally orientable surface such that if we cut the graph from the surface, the remaining part consists of connected components called faces or cells, each homeomorphic to an open disk. The map can also be represented (not in a unique way) as a ribbon graph on the plane keeping the incidence order of the edges around each vertex. In such a representation, two half-edges can be parallel or cross in the middle. We say that the hypermap is orientable if it is embedded in an orientable surface (sphere, torus, bretzel, ...). Otherwise the hypermap is embedded in a non orientable surface (projective plane, Klein bottle, ...) and is said to be non orientable. More details about hypermaps can be found in [20].

Hypermaps are usually classified according to a triple of integer partitions that give respectively the degree distribution of the faces, the degree distribution of the white vertices, and the degree distribution of the black vertices. For any integer $n$ and partitions $\lambda, \mu$ and $\nu$ of $n$, denote $l_{\mu, \nu}^{\lambda}$ (resp. $m_{\mu, \nu}^{\lambda}$ ) the number of locally orientable hypermaps (resp. orientable) of face degree distribution $\lambda$, white vertices degree distribution $\mu$ and black vertices degree distribution $\nu$. In the general case, the generating functions for the $l_{\mu, \nu}^{\lambda}$ (resp. $m_{\mu, \nu}^{\lambda}$ ) and the generating functions of the connection coefficients $\tilde{b}_{\mu, \nu}^{\lambda}$ (resp. $c_{\mu, \nu}^{\lambda}$ ) are connected through Equation (2) of [8]. When of the three integer partitions is equal to the one part partition $(n)$ (say $\mu=(n)$ ) then the coefficients are equal. Namely:

$$
\begin{aligned}
& l_{n, \nu}^{\lambda}=\tilde{b}_{n, \nu}^{\lambda}=a_{n, \nu}^{\lambda}(2), \\
& m_{n, \nu}^{\lambda}=c_{n, \nu}^{\lambda}=a_{n, \nu}^{\lambda}(1) .
\end{aligned}
$$

This provides an additional motivation to the study of the coefficients $a_{n n}^{\lambda}(\alpha)$ as in the special case $\alpha=2, a_{n n}^{\lambda}(2)$ is the number of locally oriented hypermaps with one white and one black vertex and $\ell(\lambda)$ faces of degree distribution $\lambda$. For $\alpha=1 a_{n n}^{\lambda}(1)$ counts the number of such maps that are orientable.

Finally note that in that case (one of the partition is equal to $(n)$ ) the bipartite condition in the graph representation of the connection coefficients is equivalent to the condition of orientability of the considered hypermaps.

Example 3. Two hypermaps with one white and one black vertex are depicted on Figure 4. The leftmost (resp. rightmost) one is orientable (resp. non orientable) and has a face degree distribution $\lambda=(3,1)$ (resp. $\lambda=(3)$ ). 

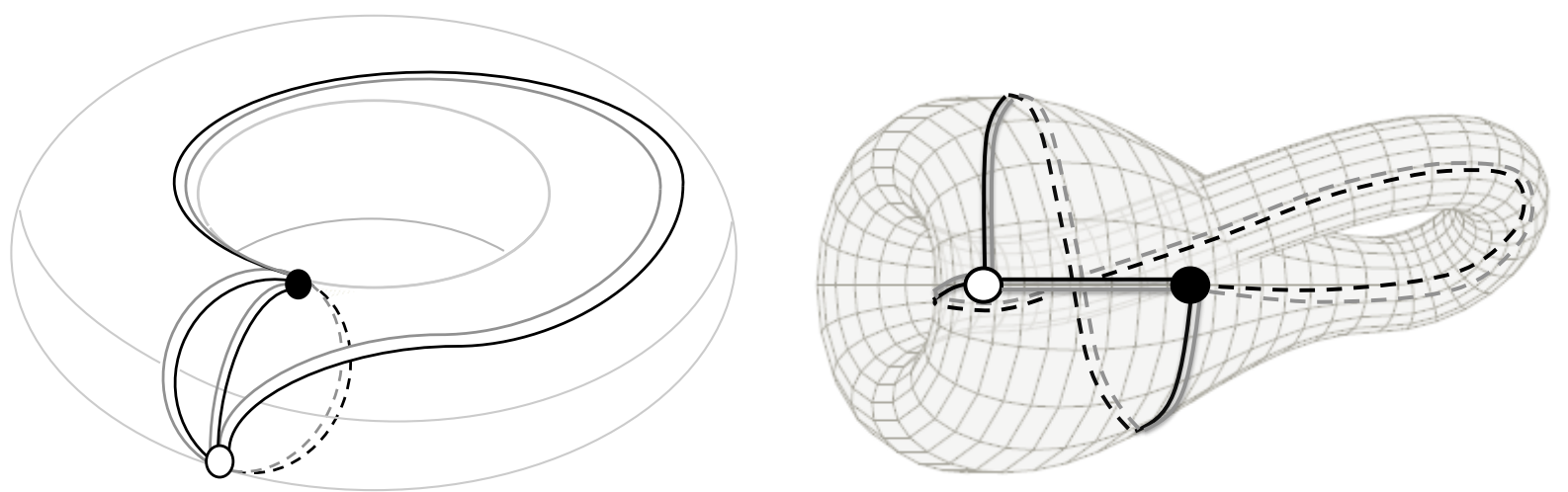

Figure 4: Examples of hypermaps with one white and one black vertex embedded in the torus (left) and the Klein bottle (right).

\section{Proof of Theorem 1}

\subsection{Laplace-Beltrami operator, generalized binomial coefficients and Pieri formula}

In order to prove Theorem 1 for general $\alpha$ we need to recall a few properties about Jack symmetric functions.

For indeterminate $x=\left(x_{1}, x_{2}, \ldots\right)$ define the Laplace-Beltrami operator by

$$
D(\alpha)=\frac{\alpha}{2} \sum_{i} x_{i}^{2} \frac{\partial^{2}}{\partial x_{i}^{2}}+\sum_{i \neq j} \frac{x_{i} x_{j}}{x_{i}-x_{j}} \frac{\partial}{\partial x_{i}} .
$$

First we have the following classical property.

Lemma 1 ([26]). The Jack's symmetric functions are eigenfunctions of $D(\alpha)$ :

$$
D(\alpha) J_{\lambda}^{\alpha}=\theta_{\left[1^{|\lambda|-2} 2^{1}\right]}^{\lambda}(\alpha) J_{\lambda}^{\alpha}
$$

Then, for two partitions, $\mu \subseteq \lambda$ the generalised binomial coefficients $\left(\begin{array}{l}\lambda \\ \mu\end{array}\right)$ are defined through the relation

$$
\frac{J_{\lambda}^{\alpha}\left(1+x_{1}, 1+x_{2}, \ldots\right)}{J_{\lambda}^{\alpha}(1,1, \ldots)}=\sum_{\mu \subseteq \lambda}\left(\begin{array}{l}
\lambda \\
\mu
\end{array}\right) \frac{J_{\mu}^{\alpha}\left(x_{1}, x_{2}, \ldots\right)}{J_{\mu}^{\alpha}(1,1, \ldots)} .
$$

Details about the existence and properties of these binomial coefficients can be found in $[21,22]$. As shown in [29] these coefficients are equal to some properly normalised shifted Jack polynomials. For $\rho \vdash n+1$ and integer $1 \leqslant i \leqslant \ell(\rho)$ define the partition $\rho_{(i)}$ of $n$ (if it exists) obtained by replacing $\rho_{i}$ in $\rho$ by $\rho_{i}-1$ and keeping all the other parts as in $\rho$. Similarly for $\gamma \vdash n$ and integer $1 \leqslant i \leqslant \ell(\gamma)+1$ we define the partition $\gamma^{(i)}$ of $n+1$ (if 
it exists) obtained by replacing $\gamma_{i}$ in $\gamma$ by $\gamma_{i}+1$ and keeping all the other parts as in $\gamma$. Define also the numbers $c_{i}(\gamma)$ as

$$
c_{i}(\gamma)=\alpha\left(\begin{array}{c}
\gamma^{(i)} \\
\gamma
\end{array}\right) \frac{j_{\gamma}(\alpha)}{j_{\gamma^{(i)}}(\alpha)} .
$$

As a result of this definition, one has:

$$
c_{i}\left(\rho_{(i)}\right)=\alpha\left(\begin{array}{c}
\rho \\
\rho_{(i)}
\end{array}\right) \frac{j_{\rho_{(i)}}(\alpha)}{j_{\rho}(\alpha)} .
$$

In [21] Lassalle showed that the Pieri formula for Jack symmetric functions can be written as

$$
p_{1} J_{\gamma}^{\alpha}=\sum_{i=1}^{\ell(\gamma)+1} c_{i}(\gamma) J_{\gamma^{(i)}}^{\alpha}
$$

Additionally, denote $p_{1}^{\perp}$ the conjugate operator of $p_{1}$ with respect to the scalar product $\langle\cdot, \cdot\rangle_{\alpha}$. Lassalle showed [21] that

$$
p_{1}^{\perp} J_{\rho}^{\alpha}=\alpha \frac{\partial}{\partial p_{1}} J_{\rho}^{\alpha}=\alpha \sum_{i=1}^{\ell(\rho)}\left(\begin{array}{c}
\rho \\
\rho_{(i)}
\end{array}\right) J_{\rho_{(i)}}^{\alpha} .
$$

Following [23], we introduce the two conjugate operators $E_{2}$ and $E_{2}^{\perp}$

$$
\begin{aligned}
& E_{2}=\left[D(\alpha), p_{1} / \alpha\right]=\sum_{k \geqslant 1} k p_{k+1} \frac{\partial}{\partial p_{k}}, \\
& E_{2}^{\perp}=\left[p_{1}^{\perp} / \alpha, D(\alpha)\right]=\sum_{k \geqslant 1}(k+1) p_{k} \frac{\partial}{\partial p_{k+1}} .
\end{aligned}
$$

Using indeterminate $x=\left(x_{1}, x_{2}, \ldots\right)$, one can express $E_{2}$ (see [23]) as:

$$
E_{2}=\sum_{i} x_{i}^{2} \frac{\partial}{\partial x_{i}}
$$

\subsection{Relating two series for Jack symmetric functions}

We are now ready to show our main formula. Our general strategy relies on known Equation (24) that establishes a recurrence like relation between Jack symmetric function indexed by integer partitions $n$ and $n+1$ and the known results about the LaplaceBeltrami operator, including Equation (26).

As the first step this section is devoted to the proof of the following theorem.

Theorem 5. Let $x$ and $y$ be two indeterminates. The following relation holds:

$$
\sum_{\rho \vdash n+1} \frac{\theta_{n+1}^{\rho}(\alpha) J_{\rho}^{\alpha}(x) E_{2}^{\perp} J_{\rho}^{\alpha}(y)}{j_{\rho}(\alpha)}=\sum_{\gamma \vdash n} \frac{\theta_{n}^{\gamma}(\alpha) J_{\gamma}^{\alpha}(y)\left[D(\alpha), E_{2}\right] J_{\gamma}^{\alpha}(x)}{j_{\gamma}(\alpha)} .
$$


From Equation (25), (23) and (21), one gets for any integer partition $\rho \vdash n+1$

$$
\begin{aligned}
\frac{E_{2}^{\perp} J_{\rho}^{\alpha}(y)}{j_{\rho}(\alpha)} & =\frac{1}{j_{\rho}(\alpha)}\left[p_{1}^{\perp} / \alpha, D(\alpha)\right] J_{\rho}^{\alpha}(y) \\
& =\frac{1}{\alpha j_{\rho}(\alpha)} p_{1}^{\perp} D(\alpha) J_{\rho}^{\alpha}(y)-\frac{1}{\alpha} D(\alpha)\left(\sum_{i=1}^{\ell(\rho)} c_{i}\left(\rho_{(i)}\right) \frac{J_{\rho_{(i)}}^{\alpha}(y)}{j_{\rho_{(i)}}(\alpha)}\right) \\
& =\frac{1}{\alpha} \sum_{i=1}^{\ell(\rho)} c_{i}\left(\rho_{(i)}\right)\left(\theta_{\left[1^{n-1} 2\right]}^{\rho}(\alpha)-\theta_{\left[1^{n-2} 2\right]}^{\rho_{(i)}}(\alpha)\right) \frac{J_{\rho_{(i)}}^{\alpha}(y)}{j_{\rho_{(i)}}(\alpha)} .
\end{aligned}
$$

Multiplying both sides by $\theta_{n+1}^{\rho}(\alpha) J_{\rho}^{\alpha}(x)$ and summing over all partitions $\rho$ of $n+1$ yields

$$
\begin{aligned}
& \sum_{\rho \vdash n+1} \frac{\theta_{n+1}^{\rho}(\alpha) J_{\rho}^{\alpha}(x) E_{2}^{\perp} J_{\rho}^{\alpha}(y)}{j_{\rho}(\alpha)}= \\
& \frac{1}{\alpha} \sum_{\rho \vdash n+1} \sum_{i=1}^{\ell(\rho)} c_{i}\left(\rho_{(i)}\right)\left(\theta_{\left[1^{n-1} 2\right]}^{\rho}(\alpha)-\theta_{\left[1^{n-2} 2\right]}^{\rho_{(i)}}(\alpha)\right) \frac{\theta_{n+1}^{\rho}(\alpha) J_{\rho}^{\alpha}(x) J_{\rho_{(i)}}^{\alpha}(y)}{j_{\rho(i)}(\alpha)} .
\end{aligned}
$$

Reorganizing the summation indices on the right-hand side gives

$$
\begin{aligned}
& \sum_{\rho \vdash n+1} \frac{\theta_{n+1}^{\rho}(\alpha) J_{\rho}^{\alpha}(x) E_{2}^{\perp} J_{\rho}^{\alpha}(y)}{j_{\rho}(\alpha)}= \\
& \frac{1}{\alpha} \sum_{\gamma \vdash n} \sum_{i=1}^{\ell(\gamma)+1} c_{i}(\gamma)\left(\theta_{\left[1^{\left.\gamma^{n-1} 2\right]}\right.}^{(i)}(\alpha)-\theta_{\left[1^{n-2} 2\right]}^{\gamma}(\alpha)\right) \frac{\theta_{n+1}^{\gamma^{(i)}}(\alpha) J_{\gamma^{(i)}}^{\alpha}(x) J_{\gamma}^{\alpha}(y)}{j_{\gamma}(\alpha)} .
\end{aligned}
$$

We prove the following lemma.

Lemma 2. For $\gamma \vdash n$ and integer $i$ the following relation is fulfilled:

$$
\theta_{n+1}^{\gamma^{(i)}}(\alpha)=\theta_{n}^{\gamma}(\alpha)\left(\theta_{\left[1^{n-1} 2\right]}^{\gamma^{(i)}}(\alpha)-\theta_{\left[1^{n-2} 2\right]}^{\gamma}(\alpha)\right)
$$

Proof. According to Equations (18) and (17)

$$
\begin{aligned}
& \theta_{n+1}^{\gamma^{(i)}}(\alpha)=\prod_{s \in \gamma^{(i)} \backslash(1,1)}\left(\alpha a^{\prime}(s)-l^{\prime}(s)\right), \\
& \theta_{\left[1^{n-1} 2\right]}^{\gamma^{(i)}}(\alpha)=\sum_{s \in \gamma^{(i)}}\left(\alpha a^{\prime}(s)-l^{\prime}(s)\right) .
\end{aligned}
$$

As per its definition, the Young diagram of $\gamma^{(i)}$ is obtained from the one of $\gamma$ by adding a box in the position $\left(i, \gamma_{i}+1\right)$. But $a^{\prime}(s)$ and $l^{\prime}(s)$ are only functions of the number of boxes to the west and the north of the considered square $s$. As a result the value of 
$\left(\alpha a^{\prime}(s)-l^{\prime}(s)\right)$ for a given $s$ in $\gamma^{(i)}$ is the same as for the corresponding box in $\gamma$ except for the special case $s=\left(i, \gamma_{i}+1\right)$. Therefore

$$
\begin{aligned}
\theta_{n+1}^{\gamma^{(i)}}(\alpha) & =\left(\prod_{s \in \gamma \backslash(1,1)}\left(\alpha a^{\prime}(s)-l^{\prime}(s)\right)\right)\left(\alpha a^{\prime}\left(i, \gamma_{i}+1\right)-l^{\prime}\left(i, \gamma_{i}+1\right)\right) \\
& =\theta_{n}^{\gamma}(\alpha)\left(\theta_{\left[1^{n-1} 2\right]}^{\gamma^{(i)}}(\alpha)-\theta_{\left[1^{n-2} 2\right]}^{\gamma}(\alpha)\right) .
\end{aligned}
$$

This is the desired formula.

Thanks to Lemma 2 we get

$$
\begin{aligned}
& \sum_{\rho \vdash n+1} \frac{\theta_{n+1}^{\rho}(\alpha) J_{\rho}^{\alpha}(x) E_{2}^{\perp} J_{\rho}^{\alpha}(y)}{j_{\rho}(\alpha)}= \\
& \frac{1}{\alpha} \sum_{\gamma \vdash n} \frac{\theta_{n}^{\gamma}(\alpha) J_{\gamma}^{\alpha}(y)}{j_{\gamma}(\alpha)} \sum_{i=1}^{\ell(\gamma)+1} c_{i}(\gamma)\left(\theta_{\left[1^{n-1} 2\right]}^{\gamma^{(i)}}(\alpha)-\theta_{\left[1^{n-2} 2\right]}^{\gamma}(\alpha)\right)^{2} J_{\gamma^{(i)}}^{\alpha}(x) .
\end{aligned}
$$

Finally, notice that the following relation holds for integers $a, b$.

$$
\begin{aligned}
\sum_{i=1}^{\ell(\gamma)+1} c_{i}(\gamma)\left(\theta_{\left[1^{n-1} 2\right]}^{\gamma^{(i)}}(\alpha)\right)^{a} & \left(\theta_{\left[1^{n-2} 2\right]}^{\gamma}(\alpha)\right)^{b} J_{\gamma^{(i)}}^{\alpha}(x) \\
& =D(\alpha)^{a}\left(\theta_{\left[1^{n-2} 2\right]}^{\gamma}(\alpha)\right)^{b} \sum_{i=1}^{\ell(\gamma)+1} c_{i}(\gamma) J_{\gamma^{(i)}}^{\alpha}(x) \\
& =D(\alpha)^{a}\left(\theta_{\left[1^{n-2} 2\right]}^{\gamma}(\alpha)\right)^{b} p_{1} J_{\gamma}^{\alpha}(x) \\
& =D(\alpha)^{a} p_{1} D(\alpha)^{b} J_{\gamma}^{\alpha}(x)
\end{aligned}
$$

Using this relation in Equation (29) gives the desired result. Indeed

$$
\begin{aligned}
\sum_{\rho \vdash n+1} & \frac{\theta_{n+1}^{\rho}(\alpha) J_{\rho}^{\alpha}(x) E_{2}^{\perp} J_{\rho}^{\alpha}(y)}{j_{\rho}(\alpha)} \\
& =\frac{1}{\alpha} \sum_{\gamma \vdash n} \frac{\theta_{n}^{\gamma}(\alpha) J_{\gamma}^{\alpha}(y)}{j_{\gamma}(\alpha)}\left(D(\alpha)^{2} p_{1}-2 D(\alpha) p_{1} D(\alpha)+p_{1} D(\alpha)^{2}\right) J_{\gamma}^{\alpha}(x) \\
& =\sum_{\gamma \vdash n} \frac{\theta_{n}^{\gamma}(\alpha) J_{\gamma}^{\alpha}(y)}{j_{\gamma}(\alpha)}\left[D(\alpha),\left[D(\alpha), p_{1} / \alpha\right]\right] J_{\gamma}^{\alpha}(x) \\
& =\sum_{\gamma \vdash n} \frac{\theta_{n}^{\gamma}(\alpha) J_{\gamma}^{\alpha}(y)}{j_{\gamma}(\alpha)}\left[D(\alpha), E_{2}\right] J_{\gamma}^{\alpha}(x) .
\end{aligned}
$$




\subsection{Recurrence relation between connection coefficients}

For $\lambda \vdash n+1$ and $\nu \vdash n$ extracting the coefficient in $p_{\lambda}(x) p_{\nu}(y)$ in Theorem 5 yields

$$
\begin{aligned}
\sum_{i: m_{i-1}(\nu) \neq 0} i\left(m_{i}(\nu)+1\right) \sum_{\rho \vdash n+1} \frac{\theta_{n+1}^{\rho}(\alpha) \theta_{\nu \uparrow(i-1)}^{\rho}(\alpha) \theta_{\lambda}^{\rho}(\alpha)}{j_{\rho}(\alpha)}= \\
\sum_{\gamma \vdash n} \frac{\theta_{n}^{\gamma}(\alpha) \theta_{\nu}^{\gamma}(\alpha)}{j_{\gamma}(\alpha)}\left[p_{\lambda}\right]\left(\left[D(\alpha), E_{2}\right] J_{\gamma}^{\alpha}\right) .
\end{aligned}
$$

According to Equation (19), we get the Jack connection coefficients $a_{n+1, \nu^{\uparrow(i-1)}}^{\lambda}(\alpha)$ multiplying the left-hand side by $\alpha^{\ell(\lambda)} z_{\lambda}$.

$$
\sum_{i: m_{i-1}(\nu) \neq 0} i\left(m_{i}(\nu)+1\right) a_{n+1, \nu^{\uparrow(i-1)}}^{\lambda}(\alpha)=\alpha^{\ell(\lambda)} z_{\lambda} \sum_{\gamma \vdash n} \frac{\theta_{n}^{\gamma}(\alpha) \theta_{\nu}^{\gamma}(\alpha)}{j_{\gamma}(\alpha)}\left[p_{\lambda}\right]\left(\left[D(\alpha), E_{2}\right] J_{\gamma}^{\alpha}\right) .
$$

The final step is to compute the remaining non explicit part $\left[p_{\lambda}\right]\left(\left[D(\alpha), E_{2}\right] J_{\gamma}^{\alpha}\right)$. We show the following lemma:

Lemma 3. The Lie hook of operators $D(\alpha)$ and $E_{2}$ is given by

$$
\begin{aligned}
{\left[D(\alpha), E_{2}\right]=(\alpha-1) \sum_{i \geqslant 1}(i-1)^{2} p_{i} \frac{\partial}{\partial p_{i-1}} } & +\sum_{i, j \geqslant 1}(i+j-1) p_{i} p_{j} \frac{\partial}{\partial p_{i+j-1}} \\
& +\alpha \sum_{i, j \geqslant 1} i j p_{i+j+1} \frac{\partial}{\partial p_{i}} \frac{\partial}{\partial p_{j}} .
\end{aligned}
$$

Proof. Recall Equations (20) and (27). We have

$$
\begin{aligned}
{\left[D(\alpha), E_{2}\right] } & =\frac{\alpha}{2} \sum_{i}\left[x_{i}^{2} \frac{\partial^{2}}{\partial x_{i}^{2}}, x_{i}^{2} \frac{\partial}{\partial x_{i}}\right]+\sum_{i \neq j}\left[\frac{x_{i} x_{j}}{x_{i}-x_{j}} \frac{\partial}{\partial x_{i}}, x_{i}^{2} \frac{\partial}{\partial x_{i}}+x_{j}^{2} \frac{\partial}{\partial x_{j}}\right] \\
& =\frac{\alpha}{2} \sum_{i}\left(2 x_{i}^{3} \frac{\partial^{2}}{\partial x_{i}^{2}}+2 x_{i}^{2} \frac{\partial}{\partial x_{i}}\right)+\sum_{i \neq j} \frac{2 x_{i}^{2} x_{j}}{x_{i}-x_{j}} \frac{\partial}{\partial x_{i}}
\end{aligned}
$$

Then, for any integer partition $\lambda$, one can easily check:

$$
\begin{aligned}
\sum_{i} x_{i}^{3} \frac{\partial^{2}}{\partial x_{i}^{2}} p_{\lambda}=\sum_{k=1}^{\ell(\lambda)} \lambda_{k}\left(\lambda_{k}-1\right) \frac{p_{\lambda_{k}+1}}{p_{\lambda_{k}}} p_{\lambda}+\sum_{k \neq l} \lambda_{k} \lambda_{l} \frac{p_{\lambda_{k}+\lambda_{l}+1}}{p_{\lambda_{k}} p_{\lambda_{l}}} p_{\lambda}, \\
\sum_{i} x_{i}^{2} \frac{\partial}{\partial x_{i}} p_{\lambda}=\sum_{k=1}^{\ell(\lambda)} \lambda_{k} \frac{p_{\lambda_{k}+1}}{p_{\lambda_{k}}} p_{\lambda} .
\end{aligned}
$$


As a next step we compute

$$
\begin{aligned}
\sum_{i \neq j} \frac{2 x_{i}^{2} x_{j}}{x_{i}-x_{j}} \frac{\partial}{\partial x_{i}} p_{\lambda} & =\sum_{k=1}^{\ell(\lambda)} \frac{p_{\lambda}}{p_{\lambda_{k}}} 2 \lambda_{k} \sum_{i \neq j} \frac{x_{i}^{\lambda_{k}+1} x_{j}}{x_{i}-x_{j}} \\
& =\sum_{k=1}^{\ell(\lambda)} \frac{p_{\lambda}}{p_{\lambda_{k}}} \lambda_{k} \sum_{i \neq j} \frac{x_{i}^{\lambda_{k}+1} x_{j}}{x_{i}-x_{j}}+\frac{x_{j}^{\lambda_{k}+1} x_{l}}{x_{j}-x_{i}} \\
& =\sum_{k=1}^{\ell(\lambda)} \frac{p_{\lambda}}{p_{\lambda_{k}}} \lambda_{k} \sum_{i \neq j} x_{i} x_{j} \frac{x_{i}^{\lambda_{k}}-x_{j}^{\lambda_{k}}}{x_{i}-x_{j}} \\
& =\sum_{k=1}^{\ell(\lambda)} \frac{p_{\lambda}}{p_{\lambda_{k}}} \lambda_{k} \sum_{i \neq j} \sum_{l=1}^{\lambda_{k}} x_{i}^{\lambda_{k}+1-l} x_{j}^{l} \\
& =\sum_{k=1}^{\ell(\lambda)} \frac{p_{\lambda}}{p_{\lambda_{k}}} \lambda_{k}\left(\sum_{l=1}^{\lambda_{k}}\left(\sum_{i} x_{i}^{\lambda_{k}+1-l} \sum_{j} x_{j}^{l}\right)-\sum_{l=1}^{\lambda_{k}} x_{i}^{\lambda_{k}+1-l} x_{i}^{l}\right) \\
& =\sum_{k=1}^{\ell(\lambda)} \frac{p_{\lambda}}{p_{\lambda_{k}}} \lambda_{k}\left(\sum_{l=1}^{\lambda_{k}} p_{\lambda_{k}+1-l} p_{l}-\lambda_{k} p_{\lambda_{k}+1}\right) .
\end{aligned}
$$

As a result, for any partition $\lambda$

$$
\sum_{i \neq j} \frac{2 x_{i}^{2} x_{j}}{x_{i}-x_{j}} \frac{\partial}{\partial x_{i}} p_{\lambda}=\sum_{k=1}^{\ell(\lambda)} \lambda_{k} \sum_{l=1}^{\lambda_{k}} \frac{p_{\lambda_{k}+1-l} p_{l}}{p_{\lambda_{k}}} p_{\lambda}-\sum_{k=1}^{\ell(\lambda)} \lambda_{k}^{2} \frac{p_{\lambda_{k}+1}}{p_{\lambda_{k}}} p_{\lambda} .
$$

Combining Equations (32), (33) and (34) gives the desired result.

Using this lemma we can derive

$$
\begin{aligned}
& {\left[p_{\lambda}\right]\left(\left[D(\alpha), E_{2}\right] J_{\gamma}^{\alpha}\right)=(\alpha-1) \sum_{i}(i-1)^{2}\left(m_{i-1}(\lambda)+1\right) \theta_{\lambda_{\downarrow}(i)}^{\gamma}(\alpha)} \\
& \quad+\sum_{i, j}(i+j-1)\left(m_{i+j-1}(\lambda)+1\right) \theta_{\lambda_{\downarrow}}^{\gamma}(\alpha) \\
& \quad+\alpha \sum_{i, d}(i-d-1) d\left(m_{i-d-1}(\lambda)+1\right)\left(m_{d}(\lambda)+1+\delta_{i-d-1, d}\right) \theta_{\lambda \uparrow(i-d-1, d)}^{\gamma}(\alpha) .
\end{aligned}
$$

But

$$
\begin{aligned}
& \left.\alpha^{\ell(\lambda)} z_{\lambda}(i-1)^{2}\left(m_{i-1}(\lambda)+1\right)=(i-1) i m_{i}(\lambda) z_{\lambda_{\downarrow}(i)} \alpha^{\ell\left(\lambda_{\downarrow}(i)\right.}\right) \\
& \alpha^{\ell(\lambda)} z_{\lambda} \alpha(i-d-1) d\left(m_{i-d-1}(\lambda)+1\right)\left(m_{d}(\lambda)+1+\delta_{i-d-1, d}\right) \\
& \quad=i m_{i}(\lambda) z_{\lambda^{\uparrow}(i-d-1, d)} \alpha^{\ell\left(\lambda^{\uparrow(i-d-1, d)}\right)}, \\
& \left.\alpha^{\ell(\lambda)} z_{\lambda}(i+j-1)\left(m_{i+j-1}(\lambda)+1\right)=\operatorname{\alpha ijm}_{i}(\lambda)\left(m_{j}(\lambda)-\delta_{i j}\right) z_{\lambda_{\downarrow}(i, j)} \alpha^{\ell\left(\lambda_{\downarrow}(i, j)\right.}\right) .
\end{aligned}
$$


As a result

$$
\begin{aligned}
\alpha^{\ell(\lambda)} z_{\lambda}\left[p_{\lambda}\right]\left(\left[D(\alpha), E_{2}\right] J_{\gamma}^{\alpha}\right) & =\sum_{i} i m_{i}(\lambda)\left[(\alpha-1)(i-1) \alpha^{\ell\left(\lambda_{\downarrow(i)}\right)} z_{\lambda_{\downarrow}(i)} \theta_{\lambda_{\downarrow}(i)}^{\gamma}(\alpha)\right. \\
& +\sum_{d=1}^{i-2} z_{\lambda^{\uparrow(i-1-d, d)}} \alpha^{\ell\left(\lambda^{\uparrow(i-1-d, d)}\right)} \theta_{\lambda^{\uparrow(i-1-d, d)}}^{\gamma}(\alpha) \\
& \left.+\alpha \sum_{j} j\left(m_{j}(\lambda)-\delta_{i j}\right) z_{\lambda_{\downarrow}(i, j)} \alpha^{\ell\left(\lambda_{\downarrow(i, j)}\right)} \theta_{\lambda_{\downarrow}}^{\gamma}(\alpha)\right] .
\end{aligned}
$$

The substitution of the above value for $\alpha^{\ell(\lambda)} z_{\lambda}\left[p_{\lambda}\right]\left(\left[D(\alpha), E_{2}\right] J_{\gamma}^{\alpha}\right)$ in Equation (31) gives the desired relation.

\section{Proof of the Matchings-Jack conjecture}

\subsection{Proof of Theorem 2}

In order to prove Theorem 2 we need to show that

$$
(\alpha-1)\left(\lambda_{i}-1\right) a_{n n}^{\lambda_{\downarrow}\left(\lambda_{i}\right)}(\alpha)+\sum_{d=1}^{\lambda_{i}-2} a_{n n}^{\lambda^{\uparrow\left(\lambda_{i}-1-d, d\right)}}(\alpha)+\alpha \sum_{j \neq i} \lambda_{j} a_{n n}^{\lambda_{\downarrow}\left(\lambda_{i}, \lambda_{j}\right)}(\alpha)
$$

is independent of the choice of integer $i \in\{1, \ldots, \ell(\lambda)\}$.

We introduce a few more notations. Consider the formal vector space over the polynomials in $\alpha$ spanned by the set $\left\{a_{n n}^{\lambda}(\alpha)\right\}_{n \geqslant 1, \lambda \vdash n}$. We assume that Equation (8) holds for $\nu=(n)$. For integers $i, d(i \geqslant d+2)$ and $j$ define the linear operators $\Xi_{i}, \Omega_{i, d}$ and $\Phi_{i, j}$ on the $a_{n n}^{\lambda}(\alpha)$ for all partitions $\lambda$ such that $i, j \in \lambda$ by:

$$
\begin{aligned}
& \Xi_{i} a_{n n}^{\lambda}(\alpha)=a_{n-1, n-1}^{\lambda_{\downarrow}}(\alpha), \\
& \Omega_{i, d} a_{n n}^{\lambda}(\alpha)=a_{n-1, n-1}^{\lambda^{\uparrow(i-1-d)}}(\alpha), \\
& \Phi_{i, j} a_{n n}^{\lambda}(\alpha)=a_{n-1, n-1}^{\lambda_{\downarrow(i, j)}}(\alpha) .
\end{aligned}
$$

Let also for $i \in \lambda$

$$
\Theta_{i} a_{n n}^{\lambda}(\alpha)=\left((\alpha-1)(i-1) \Xi_{i}+\sum_{d=1}^{i-2} \Omega_{i, d}+\alpha \sum_{j} j\left(m_{j}(\lambda)-\delta_{i j}\right) \Phi_{i, j}\right) a_{n n}^{\lambda}(\alpha) .
$$

We have the following proposition.

Proposition 2. Consider the operators defined above. For integers $i$ and $r$, the following identity is true on the subspace spanned by the $\left\{a_{n n}^{\lambda}(\alpha)\right\}_{n \geqslant 1, \lambda \vdash n, i, r \in \lambda}$

$$
\Theta_{r}\left(\Theta_{i}-\alpha r \Phi_{i, r}\right)=\Theta_{i}\left(\Theta_{r}-\alpha i \Phi_{i, r}\right)+\alpha(i-r) \Theta_{i+r-1} \Phi_{i, r}
$$


Proof. On can write $\Theta_{i}$ as $\Theta_{i}=C_{i}+\Sigma_{i}$ where

$$
C_{i}=(\alpha-1)(i-1) \Xi_{i}+\sum_{d=1}^{i-2} \Omega_{i, d}
$$

and

$$
\Sigma_{i}=\alpha \sum_{j} j\left(m_{j}(\lambda)-\delta_{i j}\right) \Phi_{i, j}
$$

when applied to $a_{n n}^{\lambda}(\alpha)$. The $\left(C_{i}\right)_{i \geqslant 1}$ commute with each other. The $\left(\Sigma_{i}\right)_{i \geqslant 1}$ do neither commute with each other nor with the $\left(C_{i}\right)_{i \geqslant 1}$. The left-hand side of Equation (35) reads:

$$
\Theta_{r}\left(\Theta_{i}-\alpha r \Phi_{i, r}\right)=\left(C_{r}+\Sigma_{r}\right)\left(C_{i}+\Sigma_{i}-\alpha r \Phi_{i, r}\right) .
$$

We use the following lemma.

Lemma 4. Let $\left(C_{i}\right)_{i \geqslant 1}$ and $\left(\Sigma_{i}\right)_{i \geqslant 1}$ be defined as above. For integers $i$ and $r C_{i}, \Sigma_{i}$ and $\Sigma_{r}$ verify

$$
\begin{aligned}
& \Sigma_{r}\left(\Sigma_{i}-\alpha r \Phi_{i, r}\right)=\Sigma_{i}\left(\Sigma_{r}-\alpha i \Phi_{i, r}\right)+\alpha(i-r) \Sigma_{i+r-1} \Phi_{i, r} \\
& \Sigma_{r} C_{i}=C_{i}\left(\Sigma_{r}-\alpha i \Phi_{i, r}\right)+\alpha\left((\alpha-1)(i-1)^{2} \Xi_{i+r-1}\right. \\
& \left.+\sum_{d=1}^{i-2}(i-1-d) \Omega_{i+r-1, d}-\sum_{d=r-1}^{i+r-3}(r-1-d) \Omega_{i+r-1, d}\right) \Phi_{i, r} .
\end{aligned}
$$

The proof of Lemma 4 uses elementary techniques and is rather cumbersome. For the sake of clarity we postpone it to Section 4.4.

Applying Equation (37) to $\Sigma_{r} C_{i}$ and to $C_{r}\left(\Sigma_{i}-\alpha r \Phi_{i, r}\right)$, yields

$$
\begin{aligned}
& \Sigma_{r} C_{i}+C_{r}\left(\Sigma_{i}-\alpha r \Phi_{i, r}\right)=C_{i}\left(\Sigma_{r}-\alpha i \Phi_{i, r}\right)+\Sigma_{i} C_{r} \\
&+\alpha\left((\alpha-1)\left[(i-1)^{2}-(r-1)^{2}\right] \Xi_{i+r-1}+\sum_{d=1}^{i+r-3}(i-r) \Omega_{i+r-1, d}\right) \Phi_{i, r} \\
&=C_{i}\left(\Sigma_{r}-\alpha i \Phi_{i, r}\right)+\Sigma_{i} C_{r}+\alpha(i-r) C_{i+r-1} \Phi_{i, r}
\end{aligned}
$$

As a result,

$$
\begin{aligned}
& \left(C_{r}+\Sigma_{r}\right)\left(C_{i}+\Sigma_{i}-\alpha r \Phi_{i, r}\right) \\
& =C_{r} C_{i}+C_{r}\left(\Sigma_{i}-\alpha r \Phi_{i, r}\right)+\Sigma_{r} C_{i}+\Sigma_{r}\left(\Sigma_{i}-\alpha r \Phi_{i, r}\right) \\
& \stackrel{(36),(38)}{=} C_{i} C_{r}+C_{i}\left(\Sigma_{r}-\alpha i \Phi_{i, r}\right)+\Sigma_{i} C_{r}+\alpha(i-r) C_{i+r-1} \Phi_{i, r} \\
& \quad+\Sigma_{i}\left(\Sigma_{r}-\alpha i \Phi_{i, r}\right)+\alpha(i-r) \Sigma_{i+r-1} \Phi_{i, r}
\end{aligned} \quad \begin{gathered}
\left.\Sigma_{i+r-1}\right) \Phi_{i, r} .
\end{gathered}
$$

This is the desired formula. 
To prove Theorem 2, we need to show the following equivalent statement.

Theorem 6. For all integers $i, r$ and partition $\lambda \vdash n$ containing $i$ and $r$

$$
\Theta_{i} a_{n n}^{\lambda}(\alpha)=\Theta_{r} a_{n n}^{\lambda}(\alpha)=a_{n n}^{\lambda}(\alpha) .
$$

Proof. By recurrence on $n$. The property is trivial for $n=2$. Assume $\Theta_{i} a_{k, k}^{\mu}(\alpha)=a_{k, k}^{\mu}(\alpha)$ for all $2 \leqslant k \leqslant n$ whenever $i \in \mu \vdash k$. Then for partition $\lambda \vdash n+1$ containing at least one part $r$ and one part $i$, we have

$$
\Theta_{i} a_{n+1, n+1}^{\lambda}(\alpha)=\left(\Theta_{i}-\alpha r \Phi_{i, r}\right) a_{n+1, n+1}^{\lambda}(\alpha)+\alpha r \Phi_{i, r} a_{n+1, n+1}^{\lambda}(\alpha) .
$$

According to the recurrence hypothesis, as $\left(\Theta_{i}-\alpha r \Phi_{i, r}\right) a_{n+1, n+1}^{\lambda}(\alpha)$ depends on the $a_{n n}^{\mu}(\alpha)$ with $r \in \mu$, we have

$$
\left(\Theta_{i}-\alpha r \Phi_{i, r}\right) a_{n+1, n+1}^{\lambda}(\alpha)=\Theta_{r}\left(\Theta_{i}-\alpha r \Phi_{i, r}\right) a_{n+1, n+1}^{\lambda}(\alpha) .
$$

Then in view of Proposition 2

$$
\begin{aligned}
\Theta_{i} a_{n+1, n+1}^{\lambda}(\alpha)=\left(\Theta_{i}\left(\Theta_{r}-\alpha i \Phi_{i, r}\right)\right. & \left.+\alpha(i-r) \Theta_{i+r-1} \Phi_{i, r}\right) a_{n+1, n+1}^{\lambda}(\alpha) \\
& +\alpha r \Phi_{i, r} a_{n+1, n+1}^{\lambda}(\alpha) .
\end{aligned}
$$

Notice that $\Phi_{i, r} a_{n+1, n+1}^{\lambda}(\alpha)=a_{n n}^{\lambda_{\downarrow(i, r)}}(\alpha)$ and $\lambda_{\downarrow(i, r)}$ contains $i+r-1$. Applying the recurrence hypothesis to $\Theta_{i}$ and $\Theta_{i+r-1}$ gives

$$
\begin{aligned}
\Theta_{i} a_{n+1, n+1}^{\lambda}(\alpha) & =\left(\left(\Theta_{r}-\alpha i \Phi_{i, r}\right)+\alpha(i-r) \Phi_{i, r}+\alpha r \Phi_{i, r}\right) a_{n+1, n+1}^{\lambda}(\alpha) \\
& =\Theta_{r} a_{n+1, n+1}^{\lambda}(\alpha) .
\end{aligned}
$$

Finally, using Equation (8) in the case $\nu=(n)$

$$
\begin{aligned}
(n+1) a_{n+1, n+1}^{\lambda}(\alpha) & =\sum_{i} i m_{i}(\lambda) \Theta_{i} a_{n+1, n+1}^{\lambda}(\alpha) \\
& =\left(\sum_{i} i m_{i}(\lambda)\right) \Theta_{r} a_{n+1, n+1}^{\lambda}(\alpha) \\
& =(n+1) \Theta_{r} a_{n+1, n+1}^{\lambda}(\alpha) .
\end{aligned}
$$

\subsection{Graph interpretation, bijective proof of Theorem 2 in the case $\alpha \in\{1,2\}$}

In this section we provide a bijective construction to show Theorem 2 for $\alpha \in\{1,2\}$ using the combinatorial interpretation of the coefficients in these cases as described by Equation (4). While Theorem 2 has been proved for general $\alpha$ in the previous section, the proposed bijection has two important aspects. On the one hand, it provides an intuitive combinatorial interpretation of our recurrence formula. On the other hand, we use it to propose a constructive proof of the Matchings-Jack conjecture later on. The recursion is obtained by removing and replacing edges in graphs. Note that this operation is also considered in [6] in terms of maps. 


\subsubsection{Reduction of $\lambda$-graphs by replacing of edges}

Consider a $\lambda$-graph $G$ consisting of cycles $L_{2 \lambda_{1}}, L_{2 \lambda_{2}}, \ldots L_{2 \lambda_{p}}$. Let $a$ and $b$ be nonneighbour vertices of $G$. Let $a_{1}, a_{2}$ be the neighbors of $a$ and $b_{1}, b_{2}$ the neighbors of $b$ such that $\left\{a, a_{1}\right\},\left\{b, b_{1}\right\}$ are gray edges and $\left\{a, a_{2}\right\},\left\{b, b_{2}\right\}$ are black edges. Replace the edges $\left\{a, a_{1}\right\}$ and $\left\{b, b_{1}\right\}$ by the gray edge $\left\{a_{1}, b_{1}\right\}$ and, similarly, replace the edges $\left\{a, a_{2}\right\}$ and $\left\{b, b_{2}\right\}$ by the black edge $\left\{a_{2}, b_{2}\right\}$. Then the $\lambda$-graph $G$ transforms into a $\lambda^{\prime}$-graph $G^{\prime}$ where

$$
\lambda^{\prime}:= \begin{cases}\lambda_{\downarrow\left(\lambda_{i}\right)} & \text { if } a, b \in L_{2 \lambda_{i}} \text { and the number of vertices } \\ & \text { between } a \text { and } b \text { is odd, } \\ \lambda^{\uparrow\left(\lambda_{i}-1-d, d\right)} & \text { if } a, b \in L_{2 \lambda_{i}} \text { and the number of vertices } \\ & \text { between } a \text { and } b \text { is equal to } 2 d-2, \\ \lambda_{\downarrow\left(\lambda_{i}, \lambda_{j}\right)} & \text { if } a \in L_{2 \lambda_{i}}, b \in L_{2 \lambda_{j}}, j \neq i .\end{cases}
$$

Figure 5 depicts the three cases with the vertices of the cycle $L_{2 \lambda_{i}}$ labelled $1, \widehat{1}, \ldots, \lambda_{i}, \widehat{\lambda_{i}}$ and $a=1$.
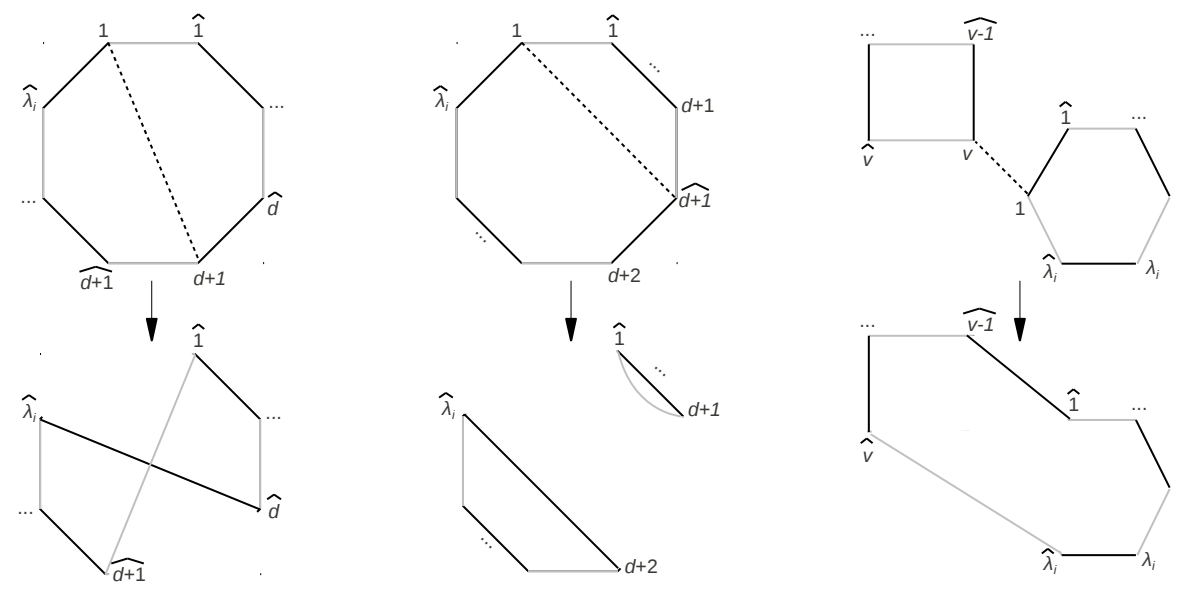

Figure 5: The replacing of edges transforms a $\lambda$-graph into a $\lambda^{\prime}$-graph (three cases).

Remark 4. These three cases correspond to classification of edges of maps considered in [6]. One can translate this terminology of classical graphs in terms of maps. Case 1 corresponds to twisted edges from [6], Case 2 corresponds to straight edges and the Case 3 corresponds to interface edges. Besides bipartite matchings correspond to the case of orientable surfaces (in particular, twisted edges are not possible in this case).

\subsubsection{Proof of Theorem 2 in the cases $\alpha \in\{1,2\}$}

One can consider that the vertices of the $2 \lambda_{i}$-cycle are labeled by elements $1, \widehat{1}, \ldots, \lambda_{i}, \widehat{\lambda_{i}}$. In order to prove Theorem 2 for $\alpha=2$ fix any vertex of the $2 \lambda_{i}$-cycle, say 1 . All $\tilde{b}_{n+1, n+1}^{\lambda}$ good matchings $\delta$ are split into three groups depending on the position of the vertex $v$ 
which is connected to 1 in $\delta$. Write $\delta=\{\{1, v\}\} \cup \delta^{\prime}$ and denote by $G^{\prime}$ a $\lambda^{\prime}$-graph obtained from $G$ by the replacing of edges described in Section 4.2.1 ( $\lambda^{\prime}$ is defined as in (39) where $a=1$ and $b=v)$. It is clear that the matching $\delta$ is good for $G$ if and only if the matching $\delta^{\prime}$ is good for $G^{\prime}$. Indeed, $(n+1)$-cycles $\mathbf{g} \cup \delta$ and $\mathbf{b} \cup \delta$ transform into $n$-cycles $\mathbf{g}^{\prime} \cup \delta^{\prime}$ and $\mathbf{b}^{\prime} \cup \delta^{\prime}$ respectively, where $\mathbf{g}^{\prime}$ and $\mathbf{b}^{\prime}$ are the sets of gray and black edges of the new $\lambda^{\prime}$-graph $G^{\prime}$. Consider three cases as above.

Case 1: $v \in\left\{2, \ldots, \lambda_{i}\right\}$ (this case is possible if $\lambda_{i} \geqslant 2$ ). As $\lambda^{\prime}=\lambda_{\downarrow\left(\lambda_{i}\right)}$ and $v$ runs over a set of $\lambda_{i}-1$ vertices in this case, then the number of such matchings $\delta$ is equal to $\left(\lambda_{i}-1\right) \tilde{b}_{n n}^{\lambda_{\downarrow(i)}}$.

Case 2: $v=\widehat{d}$ where $d \in\left\{2, \ldots, \lambda_{i}-1\right\}$ (this case is possible if $\lambda_{i} \geqslant 3$ ). In this case, $\lambda^{\prime}=\lambda^{\uparrow\left(\lambda_{i}-1-d, d\right)}$, so the number of such matchings $\delta$ for fixed $d$ is equal to $\tilde{b}_{n n}^{\lambda^{\uparrow\left(\lambda_{i}-1-d, d\right)}}$.

Case 3: $v$ belongs to the $2 \lambda_{j}$-cycle where $j \neq i$. Now $\lambda^{\prime}=\lambda_{\downarrow\left(\lambda_{i}, \lambda_{j}\right)}$ and as $v$ runs the set of $2 \lambda_{j}$ vertices then the number of such matchings $\delta$ is equal to $2 \lambda_{j} \tilde{b}_{n n}^{\lambda_{\downarrow}\left(\lambda_{i}, \lambda_{j}\right)}$.

Summing the numbers of good matchings in all cases we obtain the desired formula.

The proof in the case $\alpha=1$ is similar. As good matchings have to be bipartite, the case 1 is not possible and in the case $3, v \in[\widehat{n}]$ so the factor before $c_{n n}^{\lambda_{\downarrow}\left(\lambda_{i}, \lambda_{j}\right)}$ is equal to $\lambda_{j}$ instead of $2 \lambda_{j}$.

Remark 5. One can prove the same result in terms of permutations without using graphs. But this method is cumbersome even in the simple case of the coefficients $c_{n n}^{\lambda}$. Nevertheless, it is useful to interpret the recurrence formula in terms of permutations. Let $\sigma=\tau \rho$ be a factorisation of a permutation $\sigma \in S_{n+1}$ into the product of two $(n+1)$-cycles. Suppose that the cycle type of $\sigma$ is $\lambda$ and element $n+1$ belongs to the cycle of size $\lambda_{i}$. Denote by $\tau^{\prime}$ and $\rho^{\prime}$ the $n$-cycles obtained from $\sigma$ and $\tau$ by removing $n+1$. One can prove that the cycle type of $\sigma^{\prime} \tau^{\prime} \in S_{n}$ is $\lambda^{\uparrow\left(\lambda_{i}-1-d, d\right)}$ for some $d \in\left\{1, \ldots, \lambda_{i}-2\right\}$ or $\lambda_{\downarrow\left(\lambda_{i}, \lambda_{j}\right)}$ for some $j \neq i$.

Examples 1. Let us show that $c_{55}^{5}=2 c_{44}^{(3,1)}+c_{44}^{(2,2)}$ in terms of permutations.

1. There are three ways of writing $(123)(4) \in S_{4}$ as a product of two 4-cycles. The corresponding factorisations of $(12345) \in S_{5}$ into the product of two 5-cycles are

$$
\begin{aligned}
& (123)(4)=(1432)(1324) \leftrightarrow \quad(12345)=(15432)(13524), \\
& (123)(4)=(1324)(1342) \leftrightarrow \quad(12345)=(13254)(13542), \\
& (123)(4)=(1342)(1432) \leftrightarrow(12345)=(13542)(14352) .
\end{aligned}
$$

2. There are two ways of writing $(12)(34) \in S_{4}$ as the product of two 4-cycles. The corresponding factorisations of $(12345) \in S_{5}$ into the product of two 5-cycles are

$$
\begin{aligned}
& (12)(34)=(1324)(1324) \quad \leftrightarrow \quad(12345)=(15324)(13254), \\
& (12)(34)=(1423)(1423) \quad \leftrightarrow \quad(12345)=(14253)(14253) .
\end{aligned}
$$

So $c_{55}^{5}=2 \cdot 3+2=8$. 


\subsection{Matching Jack Conjecture in the case $\mu=\nu=(n)$}

In this section we define the functions $\left(\mathrm{wt}_{\lambda}\right)_{\lambda \vdash n}$ and prove Statements (10), (11) and (12) for all $\lambda \vdash n$ and $\delta \in \mathcal{G}(\lambda)$ by induction on $n$.

Define $\operatorname{wt}_{(1)}(\{1, \widehat{1}\})=0$. As $a_{11}^{(1)}(\beta+1)=1=\beta^{0}$ then Statements (10)-(12) obviously hold for $n=1$.

Fix some $N \geqslant 1$ and suppose that $\mathrm{wt}_{\lambda}(\delta)$ is defined for all $\lambda$ with $|\lambda| \leqslant N$ and $\delta \in \mathcal{G}(\lambda)$ in such a way that assertions (10)-(12) are true for $n \in\{1, \ldots, N\}$. Now fix some partition $\lambda \vdash N+1$, a canonically labelled $\lambda$-graph $G$ and a matching $\delta \in \mathcal{G}(\lambda)$. In order to define the value $\mathrm{wt}_{\lambda}(\delta)$ write $\delta=\{\{1, v\}\} \cup \delta^{\prime}$ and replace the edges of $G$ associated with vertices 1 and $v$ as in Section 4.2.1. We get a $\lambda^{\prime}$-graph $G_{v}^{\prime}$ where

$$
\lambda^{\prime}= \begin{cases}\lambda_{\downarrow\left(\lambda_{1}\right)} & \text { if } v \in\left\{2, \ldots, \lambda_{1}\right\}, \\ \lambda^{\uparrow\left(d, \lambda_{1}-1-d\right)} & \text { if } v=\widehat{d} \text { where } d \in\left\{1, \ldots, \lambda_{1}-2\right\}, \\ \lambda_{\downarrow\left(\lambda_{1}, \lambda_{j}\right)} & \text { if } v \in L_{2 \lambda_{j}}\left(2 \lambda_{j} \text {-cycle of } G\right) \text { where } j>1 .\end{cases}
$$

We renumber the vertices of $G_{v}^{\prime}$ by labels $[N] \cup[\widehat{N}]$ canonically. If $v \in[\widehat{N+1}]$ then the types of vertices (with/without hat) is preserved. Denote by $\mathcal{G}_{v}\left(\lambda^{\prime}\right)$ the set of good matchings of the $\lambda^{\prime}$-graph $G_{v}^{\prime}$. It is clear that if $\delta \in \mathcal{G}(\lambda)$ then $\delta^{\prime} \in \mathcal{G}_{v}\left(\lambda^{\prime}\right)$ and if $\delta$ is bipartite then $\delta^{\prime}$ is bipartite.

The value $\mathrm{wt}_{\lambda^{\prime}}\left(\delta^{\prime}\right)$ is well defined by the inductive assumption. Define

$$
\mathrm{wt}_{\lambda}(\delta):= \begin{cases}\mathrm{wt}_{\lambda^{\prime}}\left(\delta^{\prime}\right)+1 & \text { if } v \in[N+1], \\ \mathrm{wt}_{\lambda^{\prime}}\left(\delta^{\prime}\right) & \text { if } v \in[\widehat{N+1}] .\end{cases}
$$

According to this recursive definition, $\mathrm{wt}_{\lambda}(\delta)$ is an integer less or equal to $N$ and (11) is true for $n=N+1$. In order to prove the main equality (10) for $n=N+1$ we split the sum

$$
\sum_{\delta \in \mathcal{G}(\lambda)} \beta^{\mathrm{wt}_{\lambda}(\delta)}=\sum_{v \in[N+1]} \sum_{\delta^{\prime} \in \mathcal{G}_{v}\left(\lambda^{\prime}\right)} \beta^{\mathrm{wt}_{\lambda^{\prime}}\left(\delta^{\prime}\right)+1}+\sum_{v \in[\widehat{N+1}]} \sum_{\delta^{\prime} \in \mathcal{G}_{v}\left(\lambda^{\prime}\right)} \beta^{\mathrm{wt}_{\lambda^{\prime}}\left(\delta^{\prime}\right)}
$$

into four parts according to (40):

$$
\begin{aligned}
& \sum_{\delta \in \mathcal{G}(\lambda)} \beta^{\mathrm{wt}_{\lambda}(\delta)}=\left[\sum_{v=2}^{\lambda_{1}} \sum_{\delta^{\prime} \in \mathcal{G}_{v}\left(\lambda_{\downarrow}\left(\lambda_{1}\right)\right.}+\sum_{d=1}^{\lambda_{1}-2} \sum_{\delta^{\prime} \in \mathcal{G}_{\widehat{d}}\left(\lambda_{\uparrow\left(d, \lambda_{1}-1-d\right)}\right)}+\right. \\
& \left.+\sum_{j>1} \sum_{v \in L_{2 \lambda_{j}} \cap[N+1]}\left(\sum_{\delta^{\prime} \in \mathcal{G}_{v}\left(\lambda_{\downarrow}\left(\lambda_{1}, \lambda_{j}\right)\right.}+\sum_{\delta^{\prime} \in \mathcal{G}_{\widehat{v}}\left(\lambda_{\downarrow}\left(\lambda_{1}, \lambda_{j}\right)\right.}\right)\right] \beta^{\mathrm{wt}_{\lambda}(\delta)}= \\
& \stackrel{(41)}{=}\left(\lambda_{1}-1\right) \beta \sum_{\delta^{\prime} \in \mathcal{G}\left(\lambda_{\downarrow}\left(\lambda_{1}\right)\right.} \beta^{\mathrm{wt}_{\lambda_{\downarrow}\left(\lambda_{1}\right)}\left(\delta^{\prime}\right)}+\sum_{d=1}^{\lambda_{1}-2} \sum_{\delta^{\prime} \in \mathcal{G}\left(\lambda^{\uparrow\left(d, \lambda_{1}-1-d\right)}\right)} \beta^{\mathrm{wt}_{\lambda} \uparrow\left(d, \lambda_{1}-1-d\right)}{ }^{\left(\delta^{\prime}\right)}+ \\
& +\sum_{j>1} \lambda_{j}(\beta+1) \sum_{\delta^{\prime} \in \mathcal{G}\left(\lambda_{\downarrow}\left(\lambda_{1}, \lambda_{j}\right)\right.} \beta^{\mathrm{wt}_{\lambda_{\downarrow}\left(\lambda_{1}, \lambda_{j}\right)}\left(\delta^{\prime}\right)} .
\end{aligned}
$$


Using the inductive assumption to replace the sums in the right-hand side by the appropriate $a_{N, N}^{\mu}(\beta+1)$ and applying Theorem 2 proves Statement (10) for $n=N+1$. Finally, Equation (12) is easily obtained as:

$$
\begin{gathered}
{\left[\beta^{N}\right] a_{N+1, N+1}^{\lambda}(\beta+1)=\left(\lambda_{1}-1\right)\left[\beta^{N-1}\right] a_{N, N}^{\lambda_{\downarrow}\left(\lambda_{1}\right)}(\beta+1)} \\
+\sum_{j>1} \lambda_{j}\left[\beta^{N-1}\right] a_{N, N}^{\lambda_{\downarrow\left(\lambda_{1}, \lambda_{j}\right)}}(\beta+1)=\left(\lambda_{1}-1+\sum_{j>1} \lambda_{j}\right)(N-1) !=N !
\end{gathered}
$$

Remark 6. Our definition of the function $\mathrm{wt}_{\lambda}$ is based on the bijective construction. Note that the value $\mathrm{wt}_{\lambda}(\delta)$ depends on the choice of the starting vertex 1 in general if $\delta$ is not bipartite. For instance, the three non bipartite matchings of a (3)-graph (see Fig. 3) are equivalent as graphs, but one of them has weight 1 and the two others have weight 2 (depending on the choice of the starting vertex). Recompute $a_{33}^{3}(\beta+1)=2 \beta^{2}+\beta+1$ (see examples in Section 1.4).

\subsection{Annex : proof of Lemma 4}

In this section we prove Equation (36). Equation (37) is similar and left to the reader. Let $i$ be an integer and $\lambda$ be a partition of $n \geqslant 2$ containing at least one part $i$. According to the definition of $\Sigma_{i}$, we have:

$$
\begin{aligned}
& \Sigma_{r}\left(\Sigma_{i}-\alpha r \Phi_{i, r}\right) a_{n n}^{\lambda} \\
& \quad=\alpha \sum_{j} j\left(m_{j}(\lambda)-\delta_{i j}-\delta_{r j}\right) \sum_{r} a_{n-1, n-1}^{\lambda_{\downarrow(i, j)}}(\alpha) \\
& \quad=\alpha^{2} \sum_{j} j\left(m_{j}(\lambda)-\delta_{i j}-\delta_{r j}\right) \sum_{k} k\left(m_{k}\left(\lambda_{\downarrow}(i, j)-\delta_{r k}\right) a_{n-2, n-2}^{\lambda_{\downarrow(i, j) \downarrow(r, k)}}(\alpha)\right.
\end{aligned}
$$

Then, using that $\lambda_{\downarrow(i, j)}=\lambda \backslash\{i, j\} \cup\{i+j-1\}$, we get

$$
m_{k}\left(\lambda_{\downarrow(i, j)}\right)=m_{k}(\lambda)-\delta_{i k}-\delta_{j k}+\delta_{(i+j-1) k} .
$$

As a consequence

$$
\begin{aligned}
\Sigma_{r}\left(\Sigma_{i}-\alpha r \Phi_{i, r}\right) & a_{n n}^{\lambda} \\
= & \alpha^{2} \sum_{j, k} j\left(m_{j}(\lambda)-\delta_{i j}-\delta_{r j}\right) k\left(m_{k}(\lambda)-\delta_{r k}-\delta_{i k}\right) a_{n-2, n-2}^{\lambda_{\downarrow(i, j) \downarrow(r, k)}}(\alpha) \\
& -\alpha^{2} \sum_{j} j^{2}\left(m_{j}(\lambda)-\delta_{i j}-\delta_{r j}\right) a_{n-2, n-2}^{\lambda_{\downarrow(i, j) \downarrow(r, j)}}(\alpha) \\
& +\alpha^{2} \sum_{j} j(i+j-1)\left(m_{j}(\lambda)-\delta_{i j}-\delta_{r j}\right) a_{n-2, n-2}^{\lambda_{\downarrow(i, j) \downarrow(r, i+j-1)}}(\alpha)
\end{aligned}
$$

Combine the first and the second sums in the right-hand side:

$$
\begin{aligned}
\Sigma_{r}\left(\Sigma_{i}-\alpha r \Phi_{i, r}\right) a_{n n}^{\lambda} & \\
= & \alpha^{2} \sum_{j, k} j\left(m_{j}(\lambda)-\delta_{i j}-\delta_{r j}-\delta_{k j}\right) k\left(m_{k}(\lambda)-\delta_{r k}-\delta_{i k}\right) a_{n-2, n-2}^{\lambda_{\downarrow(i, j) \downarrow(r, k)}}(\alpha) \\
& +\alpha^{2} \sum_{j} j(i+j-1)\left(m_{j}(\lambda)-\delta_{i j}-\delta_{r j}\right) a_{n-2, n-2}^{\lambda_{\downarrow(i, j) \downarrow(r, i+j-1)}}(\alpha)
\end{aligned}
$$


As $m_{j}(\lambda)-\delta_{r j}-\delta_{k j}=m_{j}\left(\lambda_{\downarrow(r, k)}\right)-\delta_{(r+k-1) j}$ and $\lambda_{\downarrow(i, j) \downarrow(r, i+j-1)}=\lambda_{\downarrow(i, r) \downarrow(i+r-1, j)}$, we have

$$
\begin{aligned}
\Sigma_{r}\left(\sum_{i}-\alpha r\right. & \left.\Phi_{i, r}\right) a_{n n}^{\lambda} \\
= & \alpha^{2} \sum_{j, k} j\left(m_{j}\left(\lambda_{\downarrow(r, k)}\right)-\delta_{i j}-\delta_{(r+k-1) j}\right) k\left(m_{k}(\lambda)-\delta_{r k}-\delta_{i k}\right) a_{n-2, n-2}^{\lambda_{\downarrow \downarrow i, j) \downarrow(r, k)}}(\alpha) \\
& +\alpha^{2} \sum_{j} j(i+j-1)\left(m_{j}(\lambda)-\delta_{i j}-\delta_{r j}\right) a_{n-2, n-2}^{\lambda_{\downarrow(i, r) \downarrow(i+r-1, j)}}(\alpha) \\
= & \alpha^{2} \sum_{j, k} j\left(m_{j}\left(\lambda_{\downarrow(r, k)}\right)-\delta_{i j}\right) k\left(m_{k}(\lambda)-\delta_{r k}-\delta_{i k}\right) a_{n-2, n-2}^{\lambda_{\downarrow}(i, j) \downarrow(r, k)}(\alpha) \\
& +\alpha^{2} \sum_{j} j(i+j-1)\left(m_{j}(\lambda)-\delta_{i j}-\delta_{r j}\right) a_{n-2, n-2}^{\lambda_{\downarrow(i, r) \downarrow(i+r-1, j)}}(\alpha) \\
& -\alpha^{2} \sum_{k} k(r+k-1)\left(m_{k}(\lambda)-\delta_{r k}-\delta_{i k}\right) a_{n-2, n-2}^{\lambda_{\downarrow(i, r+k-1) \downarrow(r, k)}}(\alpha)
\end{aligned}
$$

Next, permuting the operations on $\lambda$ when applicable and combining the second and third sums in the right-hand side gives:

$$
\begin{aligned}
\Sigma_{r} & \left(\Sigma_{i}-\alpha r \Phi_{i, r}\right) a_{n n}^{\lambda} \\
\quad & \alpha^{2} \sum_{j, k} j\left(m_{j}\left(\lambda_{\downarrow(r, k)}\right)-\delta_{i j}\right) k\left(m_{k}(\lambda)-\delta_{r k}-\delta_{i k}\right) a_{n-2, n-2}^{\lambda_{\downarrow(r, k) \downarrow(i, j)}}(\alpha) \\
& +\alpha^{2} \sum_{j} j(i+j-1)\left(m_{j}(\lambda)-\delta_{i j}-\delta_{r j}\right) a_{n-2, n-2}^{\lambda_{\downarrow}(i, r) \downarrow(i+r-1, j)}(\alpha) \\
& -\alpha^{2} \sum_{k} k(r+k-1)\left(m_{k}(\lambda)-\delta_{r k}-\delta_{i k}\right) a_{n-2, n-2}^{\lambda_{\downarrow}(i, r) \downarrow(i+r-1, k)}(\alpha) \\
& =\Sigma_{i}\left(\Sigma_{r}-\alpha r \Phi_{i, r}\right) a_{n n}^{\lambda}+\alpha^{2}(i-r) \sum_{j} j\left(m_{j}(\lambda)-\delta_{i j}-\delta_{r j}\right) a_{n-2, n-2}^{\lambda_{\downarrow l}(i, r) \downarrow(i+r-1, j)}(\alpha)
\end{aligned}
$$

We finish the computation by using $m_{j}(\lambda)-\delta_{i j}-\delta_{r j}=m_{j}\left(\lambda_{\downarrow(i, r)}\right)-\delta_{(i+r-1) j}$ in the right-hand side and identifying the appropriate operators $\Sigma$ and $\Phi$ according to their definition.

$$
\begin{aligned}
& \Sigma_{r}\left(\Sigma_{i}-\alpha r \Phi_{i, r}\right) a_{n n}^{\lambda} \\
& =\Sigma_{i}\left(\Sigma_{r}-\alpha r \Phi_{i, r}\right) a_{n n}^{\lambda}+\alpha^{2}(i-r) \sum_{j} j\left(m_{j}\left(\lambda_{\downarrow(i, r)}\right)-\delta_{(i+r-1) j}\right) a_{n-2, n-2}^{\lambda_{\downarrow}(i, r) \downarrow(i+r-1, j)}(\alpha) \\
& =\Sigma_{i}\left(\Sigma_{r}-\alpha r \Phi_{i, r}\right) a_{n n}^{\lambda}+\alpha^{2}(i-r) \sum_{j} j\left(m_{j}\left(\lambda_{\downarrow(i, r)}\right)-\delta_{(i+r-1) j}\right) \Phi_{i+r-1, j} \Phi_{i, r} a_{n, n}^{\lambda}(\alpha) \\
& =\Sigma_{i}\left(\Sigma_{r}-\alpha r \Phi_{i, r}\right) a_{n n}^{\lambda}+\alpha(i-r) \Sigma_{i+r-1} \Phi_{i, r} a_{n, n}^{\lambda}(\alpha)
\end{aligned}
$$

\section{Proof of Theorem 4}

In this section we show the results in Theorem 4 for the coefficients $a_{\lambda}^{l, r}(\alpha)$ defined in Equation (13). Denote by $\Gamma_{n}^{l}$ the generating series for the coefficients $a_{\lambda}^{l, 0}(\alpha)$ defined as

$$
\Gamma_{n}^{l}=\sum_{\lambda \vdash n} \alpha^{-\ell(\lambda)} z_{\lambda}^{-1} a_{\lambda}^{l, 0}(\alpha) p_{\lambda}=\sum_{\gamma \vdash n} \frac{\left(\theta_{n}^{\gamma}(\alpha)\right)^{l}}{j_{\gamma}(\alpha)} J_{\gamma}^{\alpha} .
$$


We have the following proposition.

Proposition 3. Let $n$ and $l$ be two integers and $\Gamma_{n}^{l}$ the generating series defined in Equation (42) and $\Delta_{l}(\alpha)$ the operator defined in Theorem 4. We have

$$
\Gamma_{n+1}^{l}=\frac{1}{n+1} \Delta_{l}(\alpha)\left(\Gamma_{n}^{l}\right) .
$$

Proof. The proof is similar to the one in Section 3.2. In Equation (28) replace the multiplication by $\theta_{n+1}^{\rho}(\alpha)$ by a multiplication by $\left(\theta_{n+1}^{\rho}(\alpha)\right)^{l-1}$. We get

$$
\begin{aligned}
& \sum_{\rho \vdash n+1} \frac{\theta_{n+1}^{\rho}(\alpha)^{l-1} J_{\rho}^{\alpha}(x) E_{2}^{\perp} J_{\rho}^{\alpha}(y)}{j_{\rho}(\alpha)}= \\
& \frac{1}{\alpha} \sum_{\rho \vdash n+1} \sum_{i=1}^{\ell(\rho)} c_{i}\left(\rho_{(i)}\right)\left(\theta_{\left[1^{n-1} 2\right]}^{\rho}(\alpha)-\theta_{\left[1^{n-2} 2\right]}^{\rho_{(i)}}(\alpha)\right) \frac{\theta_{n+1}^{\rho}(\alpha)^{l-1} J_{\rho}^{\alpha}(x) J_{\rho_{(i)}}^{\alpha}(y)}{j_{\rho(i)}(\alpha)} .
\end{aligned}
$$

Then Lemma 2 is applied $l-1$ times instead of once so that Equation (29) becomes:

$$
\begin{aligned}
& \sum_{\rho \vdash n+1} \frac{\theta_{n+1}^{\rho}(\alpha)^{l-1} J_{\rho}^{\alpha}(x) E_{2}^{\perp} J_{\rho}^{\alpha}(y)}{j_{\rho}(\alpha)}= \\
& \frac{1}{\alpha} \sum_{\gamma \vdash n} \frac{\theta_{n}^{\gamma}(\alpha)^{l-1} J_{\gamma}^{\alpha}(y)}{j_{\gamma}(\alpha)} \sum_{i=1}^{\ell(\gamma)+1} c_{i}(\gamma)\left(\theta_{\left[1^{\gamma^{(i)} 2}\right]}^{(i)}(\alpha)-\theta_{\left[1^{n-2} 2\right]}^{\gamma}(\alpha)\right)^{l} J_{\gamma^{(i)}}^{\alpha}(x)= \\
& \frac{1}{\alpha} \sum_{\gamma \vdash n} \frac{\theta_{n}^{\gamma}(\alpha)^{l-1} J_{\gamma}^{\alpha}(y)}{j_{\gamma}(\alpha)} \sum_{i=1}^{\ell(\gamma)+1} c_{i}(\gamma) \sum_{k}^{l}\left(\begin{array}{l}
l \\
k
\end{array}\right) \theta_{\left[1^{\left.\gamma^{n-1} 2\right]}\right.}^{\gamma^{(i)}}(\alpha)^{k}\left(-\theta_{\left[1^{n-2} 2\right]}^{\gamma}(\alpha)\right)^{l-k} J_{\gamma^{(i)}}^{\alpha}(x)
\end{aligned}
$$

In view of Equation (30), one can rewrite the above expression as:

$$
\begin{aligned}
& \sum_{\rho \vdash n+1} \frac{\theta_{n+1}^{\rho}(\alpha)^{l-1} J_{\rho}^{\alpha}(x) E_{2}^{\perp} J_{\rho}^{\alpha}(y)}{j_{\rho}(\alpha)}= \\
& \quad \frac{1}{\alpha} \sum_{\gamma \vdash n} \frac{\theta_{n}^{\gamma}(\alpha)^{l-1} J_{\gamma}^{\alpha}(y)}{j_{\gamma}(\alpha)} \sum_{k}^{l}\left(\begin{array}{l}
l \\
k
\end{array}\right)(-1)^{l-k} D(\alpha)^{k} p_{1} D(\alpha)^{l-k} J_{\gamma}^{\alpha}(x) .
\end{aligned}
$$

To achieve the proof, notice that

$$
\frac{1}{\alpha} \sum_{k}\left(\begin{array}{l}
l \\
k
\end{array}\right)(-1)^{l-k} D(\alpha)^{k} p_{1} D(\alpha)^{l-k}=\left[D(\alpha),\left[\ldots,\left[D(\alpha), p_{1} / \alpha\right] \ldots\right]\right]=\Delta_{l}(\alpha),
$$

and extract the coefficients in $p_{n}(y)$ in Equation (43) :

$$
(n+1) \sum_{\rho \vdash n+1} \frac{\theta_{n+1}^{\rho}(\alpha)^{l} J_{\rho}^{\alpha}(x)}{j_{\rho}(\alpha)}=\sum_{\gamma \vdash n} \frac{\theta_{n}^{\gamma}(\alpha)^{l}}{j_{\gamma}(\alpha)} \Delta_{l}(\alpha) J_{\gamma}^{\alpha}(x) .
$$

This is the desired property. 
As a consequence of Property $3, \Gamma_{n}^{l}(x)$ can explicitly expressed in term of $\Gamma_{1}^{l}(x)$ as

$$
\Gamma_{n}^{l}(x)=\frac{1}{n !} \Delta_{l}(\alpha)^{n-1}\left(\Gamma_{1}^{l}(x)\right) .
$$

Furthermore, it is easy to show that $\Gamma_{1}^{l}(x)=p_{1} / \alpha$. We are now ready to write the generating series for coefficients $a_{\lambda}^{l, r}(\alpha)$ for any integers $l$ and $r$.

$$
\begin{aligned}
\sum_{\lambda} \alpha^{-\ell(\lambda)} z_{\lambda}^{-1} a_{\lambda}^{l, r}(\alpha) p_{\lambda} & =\sum_{\gamma} \frac{\left(\theta_{\left[1^{n-2} 2\right]}^{\gamma}(\alpha)\right)^{r}\left(\theta_{n}^{\gamma}(\alpha)\right)^{l}}{j_{\gamma}(\alpha)} J_{\gamma}^{\alpha} \\
& =D(\alpha)^{r}\left(\sum_{\gamma} \frac{\left(\theta_{n}^{\gamma}(\alpha)\right)^{l}}{j_{\gamma}(\alpha)} J_{\gamma}^{\alpha}\right) \\
& =\frac{1}{n !} D(\alpha)^{r} \Delta_{l}(\alpha)^{n-1}\left(p_{1} / \alpha\right) .
\end{aligned}
$$

To prove the polynomial properties of Theorem 4, we use the following classical lemma that can be found in the proof of Theorem 3.1 of [33].

Lemma 5 ([33]). Operator $D(\alpha)$ can be expressed as:

$$
D(\alpha)=(\alpha-1) N+\alpha U+S,
$$

where for $\lambda \vdash n$ :

$$
\begin{aligned}
N & =\frac{1}{2} \sum_{i} i(i-1) p_{i} \frac{\partial}{\partial p_{i}}, \\
U & =\frac{1}{2} \sum_{i, j} i j p_{i+j} \frac{\partial}{\partial p_{i}} \frac{\partial}{\partial p_{j}}, \\
S & =\frac{1}{2} \sum_{i, j}(i+j) p_{i} p_{j} \frac{\partial}{\partial p_{i+j}},
\end{aligned}
$$

Clearly, for any partition $\lambda \vdash n$, the coefficients in the power sum expansion of $N\left(p_{\lambda}\right)$, $U\left(p_{\lambda}\right)$ and $S\left(p_{\lambda}\right)$ are non negative integers. As a result for any partition $\lambda \vdash n$ the coefficients in the power sum basis of $D(\alpha)\left(p_{\lambda}\right)$ are polynomial in $\alpha$ with integer coefficients. Furthermore, $\Delta_{l}(\alpha)$ can be rewritten as

$$
\begin{aligned}
\Delta_{l}(\alpha) & =\left[D(\alpha),\left[\ldots,\left[D(\alpha), E_{2}\right] \ldots\right]\right] \\
& =\sum_{k}\left(\begin{array}{c}
l-1 \\
k
\end{array}\right)(-1)^{l-1-k} D(\alpha)^{k} E_{2} D(\alpha)^{l-1-k} .
\end{aligned}
$$

Equation (26) shows that the coefficients in the power sum expansion of $E_{2}\left(p_{\lambda}\right)$ are integers. As a consequence, the coefficients in the power sum expansion of $D(\alpha)^{r} \Delta_{l}^{n-1}(\alpha)\left(p_{1}\right)$ 
are polynomials in $\alpha$ with integer coefficients of degree at most $(n-1)(l-1)+r$ for $n \geqslant 2$. Denote

$$
\alpha^{-\ell(\lambda)} z_{\lambda}^{-1} a_{\lambda}^{l, r}(\alpha)=\frac{1}{\alpha n !} \sum_{i=0}^{(n-1)(l-1)+r} g_{i} \alpha^{i} .
$$

The coefficients $g_{i}$ are integers. Using [37, Thm. 5], we have

$$
\alpha(-\alpha)^{(l+r-1) n+r(1-n)-l-\ell(\lambda)} \sum_{i=0}^{(n-1)(l-1)+r} g_{i} \alpha^{-i}=\frac{1}{\alpha} \sum_{j=0}^{(n-1)(l-1)+r} g_{j} \alpha^{j} .
$$

Equating the coefficients in $\alpha^{j}$ yields:

$$
(-1)^{(l-1)(n-1)+r-1-\ell(\lambda)} g_{(l-1)(n-1)+r+1-\ell(\lambda)-j}=g_{j} .
$$

But the $g_{i}$ are non zero only for non negative $i$. As a result $g_{i}=0$ for $i \geqslant(l-1)(n-1)+$ $r+2-\ell(\lambda)$. We write

$$
\alpha^{-\ell(\lambda)} z_{\lambda}^{-1} a_{\lambda}^{l, r}(\alpha)=\frac{1}{\alpha n !} \sum_{i=0}^{(n-1)(l-1)+r+1-\ell(\lambda)} g_{i} \alpha^{i}
$$

Finally

$$
\left|C_{\lambda}\right| a_{\lambda}^{l, r}(\alpha)=\sum_{i=0}^{(n-1)(l-1)+r+1-\ell(\lambda)} g_{i} \alpha^{i+\ell(\lambda)-1}=\sum_{i=\ell(\lambda)-1}^{(n-1)(l-1)+r} g_{i-\ell(\lambda)+1} \alpha^{i}
$$

is a polynomial in $\alpha$ of degree at most $(n-1)(l-1)+r$. Furthermore:

$$
\left[\alpha^{i}\right]\left|C_{\lambda}\right| a_{\lambda}^{l, r}(\alpha)=(-1)^{(l-1)(n-1)+r+\ell(\lambda)-1}\left[\alpha^{(l-1)(n-1)+r+\ell(\lambda)-1-i}\right]\left|C_{\lambda}\right| a_{\lambda}^{l, r}(\alpha) .
$$

\section{Acknowledgements}

ALK thanks the LIX laboratory and the combinatorial model group for hosting him when part of this work was done. This project has been supported by the Metchnikov scholarship.

\section{References}

[1] F. Bédard and A. Goupil. The poset of conjugacy classes and decomposition of products in the symmetric group, Can. Math. Bull, 35(2):152-160, 1992.

[2] P. Biane. Nombre de factorisations d'un grand cycle, Séminaire Lotharingien de Combinatoire, 51:4, 2004.

[3] D.R.L. Brown and D.M. Jackson. A rooted map invariant, non-orientability and Jack symmetric functions J. Comb. Theory Ser. B, 97:430-452, 2007. 
[4] M. Dołęga and V. Féray. On Kerov polynomials for Jack characters, DMTCS Proceedings (FPSAC 2013), AS:539-550, 2013.

[5] M. Dołęga and V. Féray. Gaussian fluctuations of Young diagrams and structure constants of Jack characters, Duke Math. J., doi:10.1215/00127094-3449566, 89 pp., 2016.

[6] M. Dołęga, V. Féray and P. Śniady. Jack polynomials and orientability generating series of maps, Sém. Lothar. Combin., 70:Art. B70j, 50 pp., 2014.

[7] I. P. Goulden and D. M. Jackson. The combinatorial relationship between trees, cacti and certain connection coefficients for the symmetric group, European Journal of Combinatorics, 13:357-365, 1992.

[8] I. P. Goulden and D. M. Jackson. Connection coefficients, matchings, maps and combinatorial conjectures for Jack symmetric functions, Transactions of the American Mathematical Society, 348(3):873-892, 1996.

[9] A. Goupil and G. Schaeffer. Factoring n-cycles and counting maps of given genus, European Journal of Combinatorics, 19:819-834(16), 1998.

[10] J. Irving. On the number of factorizations of a full cycle, J. Comb. Theory Ser. A, 113(7):1549-1554, 2006.

[11] P. J. Hanlon. Jack symmetric functions and some combinatorial properties of Young symmetrizers, J. Comb. Theory Ser. A, 47:37-70, 1988.

[12] P. J. Hanlon. A Markov chain on the symmetric group and Jack symmetric functions, Discrete Mathematics, 99:123-140, 1992.

[13] P. J. Hanlon, R. P. Stanley and J. R. Stembridge. Some combinatorial aspects of the spectra of normally distributed random matrices, Contemp. Math., 138:151-174, 1992.

[14] A. Hurwitz. Über die Anzahl der Riemann'schen Flächen mit gegebenen Verzweigungspunkten, Math. Ann. 55:53-66, 1902.

[15] H. Jack. A class of symmetric polynomials with a parameter, Proc. R. Soc. Edinburgh (A), 69:1-18, 1970.

[16] D. M. Jackson. Some combinatorial problems associated with products of conjugacy classes of the symmetric group, J. Comb. Theory Ser. A, 49(2):363-369, 1988.

[17] F. Knop and S. Sahi. A recursion and a combinatorial formula for Jack polynomials, Inventiones Mathematicae, 128(1):9-22, 1997.

[18] M. A. Lacroix The combinatorics of the Jack parameter and the genus series for topological maps, PhD thesis, University of Waterloo, 2009.

[19] L. Lapointe and L. Vinet. A Rodrigues formula for the Jack polynomials and the Macdonald-Stanley conjecture, International Mathematics Research Notices, 1995(9):419-424, 1995.

[20] S. Lando and A.K. Zvonkin Graphs on surfaces and their applications, SpringerVerlag, 2004. 
[21] M. Lassalle. Une formule de Pieri pour les polynômes de Jack, C. R. Acad. Sci. Paris, Sér. I Math., 309:941-944, (1989).

[22] M. Lassalle. Une formule du binôme généralisée pour les polynômes de Jack, $C . R$. Acad. Sci. Paris, Sér. I Math., 310 (1990).

[23] M. Lassalle. A positivity conjecture for Jack polynomials, Math. Res. Lett., 15(4):661-681, 2008.

[24] M. Lassalle. Jack polynomials and free cumulants, Adv. Math., 222(6):2227-2269, 2009.

[25] I. Macdonald. Symmetric functions and Hall polynomials, Oxford University Press, Second Edition, 1999.

[26] I. Macdonald. Commuting differential operators and zonal spherical functions. Springer Lecture Notes, 1271:189-200, 1987.

[27] A. H. Morales and E. A. Vassilieva. Bijective enumeration of bicolored maps of given vertex degree distribution, DMTCS Proceedings (FPSAC 2009), AK:661-672, 2009.

[28] A. H. Morales and E. A. Vassilieva. Bijective evaluation of the connection coefficients of the double coset algebra, DMTCS Proceedings (FPSAC 2011), AO:681-692, 2011.

[29] A. Okounkov, G. Olshanski. Shifted Jack polynomials, binomial formula and applications, Math. Res. Lett. 4, $69-78,1997$.

[30] D. Poulalhon and G. Schaeffer. Factorizations of large cycles in the symmetric group, Discrete Math., 254:433-458, 2000.

[31] G. Schaeffer and E. A. Vassilieva. A bijective proof of Jackson's formula for the number of factorizations of a cycle, J. Comb. Theory Ser. A, 115(6):903-924, 2008.

[32] P. Śniady. Top degree of Jack characters and enumeration of maps. arXiv:1506.06361, 2015.

[33] R. P. Stanley. Some combinatorial properties of Jack symmetric functions, Advances in Mathematics, 77:76-115, 1989.

[34] R. P. Stanley. Some combinatorial aspects of the spectra of normally distributed matrices, Comtemporary Mathematics, 138:151-174, 1992.

[35] E. A. Vassilieva. Explicit monomial expansions of the generating series for connection coefficients, DMTCS Proceedings (FPSAC 2012), AR:123-134, 2012.

[36] E. A. Vassilieva. Long cycle factorizations : bijective computation in the general case, DMTCS Proceedings (FPSAC 2013), AS:1077-1088, 2013.

[37] E. A. Vassilieva. Polynomial properties of Jack connection coefficients and generalization of a result by Dénes, Journal of Algebraic Combinatorics, 42(1):51-71, 2015. 